\title{
XII. Die informellen Beziehungen
}

Deutsche Institutionen und Persönlichkeiten nahmen im Hinblick auf die Aufnahme diplomatischer Beziehungen mit Israel eine zögerliche Haltung ein und spiegelten damit die allgemeine Unschlüssigkeit der westdeutschen Öffentlichkeit in dieser Frage wider. In Israel waren die Fronten zwischen Befürwortern und Gegnern der Beziehungen zwischen beiden Staaten dagegen klarer abgesteckt.

Genau umgekehrt verhielt es sich im Bereich der informellen Beziehungen: Westdeutsche Akademiker und Intellektuelle sowie Teile der westdeutschen Öffentlichkeit brachten Israel ernsthaftes Interesse entgegen. Das Image des jungen Landes, das mit gesellschaftlichen Experimenten operierte, enorme Einwandererströme aufnahm und integrierte und die Wüste begrünte, machte Israel in intellektuellen Kreisen für eine Weile zum beliebtesten Land der westlichen Welt, auch in der Bundesrepublik. Die noch frische Erinnerung der nationalsozialistischen Herabstufung der Juden zu Untermenschen ließ das „Wunder

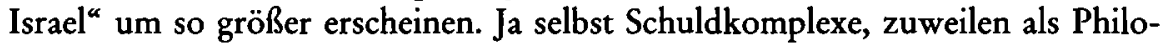
semitismus zum Ausdruck gebracht, und die Würdigung des "Jüdischen" als Weg der Wiedergutmachung - Deutschland ist bekanntlich eine Gesellschaft mit tiefen christlichen Wurzeln - förderten das Interesse an Israel noch zusätzlich. Dem wäre noch die - irrationale, romantisch verklärte - jahrhundertealte Faszination hinzuzufügen, die der Jude und das Judentum auf die westliche Zivilisation ausgeübt hat, sei es als Antichrist, als „auserwähltes Volk“, als Verkörperung des Bösen oder als Volk mit außergewöhnlichen Begabungen. Hinzu kamen schließlich schlicht Wissensdurst und Neugier, die Israel und das Judentum beim deutschen Publikum zu wecken vermochten. Aus Furcht vor arabischen Reaktionen versuchte das Auswärtige Amt, diesen proisraelischen Trend vereinzelt zu stoppen. Insgesamt begegnete ihm die westdeutsche Diplomatie jedoch mit Wohlwollen. Den Höhepunkt schien die Sympathiewelle für Israel während des Sechstagekrieges zu erreichen. Danach nahm sie mit dem Auftreten der Neuen Linken und der neuen Rolle der Palästinenser als Unterprivilegierte allmählich ab.

Solange die Holocaust-Generation bzw. die Generation der Holocaust-Überlebenden in Israel den Ton angab, demonstrierte die israelische Bevölkerung Deutschland gegenüber Kühle und Gleichgültigkeit. Dies sollte sich mit dem Heranwachsen einer neuen Generation, dem zunehmenden Gewicht des orientalischen Judentums in der israelischen Gesellschaft und der von der Mapai geförderten Zusammenarbeit mit der Bundesrepublik leicht ändern. Doch die Neugier der Israelis an Deutschland hielt sich vergleichsweise in Grenzen und war oft von latenter oder gar offener Feindseligkeit begleitet. Selbst bei den Einwanderern aus Deutschland und ihren Nachkommen mischte sich die Liebe zur deutschen Sprache und Kultur mit der Verbitterung des enttäuschten Liebhabers. 


\section{Die politische Diskussion über die kulturellen Beziehungen}

Der Religionsphilosoph Martin Buber galt als Pionier der kulturellen Beziehungen zwischen Deutschen und Juden nach dem Krieg. Er war wie durch Zufall in diese Rolle geraten, obwohl er sich anfänglich dagegen gesträubt hatte: Die Zeit sei nicht reif für Kontakte zwischen Deutschen bzw. deutschen Institutionen und ihren Partnern auf jüdischer Seite, hatte er Anfang 1952 amerikanischen Journalisten gesagt. ${ }^{1}$ Buber sprach sich gegen direkte Verhandlungen aus und weigerte sich, nach Deutschland zu reisen. ${ }^{2}$ Andererseits erklärte er sich bereit, den ihm etwa zu jener Zeit verliehenen Hansischen Goethepreis in Hamburg entgegenzunehmen. Während in den ersten Meldungen die Stadt Hamburg als Preisverleiherin angegeben wurde, stellte sich später heraus, daß es sich um eine Auszeichnung der Hamburger Universität handelte. Für Buber ein wesentlicher Unterschied: Er war bereit, quasi als Tribut an die Vorkämpfer des Humanismus einen Preis von Gelehrten und Opfern der NS-Diktatur anzunehmen, nicht jedoch von Vertretern einer Stadtverwaltung, die er als Mitläufer betrachtete. ${ }^{3}$ Seine Bereitschaft zum Empfang des Preises provozierte Verärgerung in der jüdischen Welt und entfachte eine öffentliche Debatte sowohl in Israel als auch außerhalb. Buber beharrte jedoch auf seiner Unterscheidung zwischen den Vorkämpfern des Humanismus und der passiven Bevölkerung. Anderthalb Jahre später begab er sich nach Deutschland für eine Vorlesungsreise im Auftrag der Regierung. Am 24. Juni 1953 empfing er den Hansischen Goethepreis und am 5. September desselben Jahres wurde ihm in der Paulskirche in Frankfurt am Main der Friedenspreis des Deutschen Buchhandels verliehen. In seiner Dankesrede ging er auf das jüdisch-deutsche Verhältnis bzw. auf das Verhältnis der Juden zur deutschen Kultur nach dem Holocaust ein. Dabei wiederholte er einmal mehr seine Unterscheidung zwischen jenen Deutschen, die sich zu Tausenden durch ihr grausames Verhalten selbst aus der menschlichen Sphäre ausgeschlossen hätten, und den „Kräften der Humanität ${ }^{\text {“. }}{ }^{4}$ Diese Differenzierung zwischen "guten" und „bösen“ Deutschen fand weite Verbreitung, außer in Israel, wo im Hinblick auf Deutschland weiter Pauschalurteile dominierten.

Der erste Grundsatz der israelischen Deutschlandpolitik war ein totaler Kulturboykott. Zur Begründung dieser kurz nach der Staatsgründung gefällten Entscheidung hieß es, die deutschen Institutionen seien direkt aus den Einrichtungen des NS-Regimes hervorgegangen, worüber auch der neue liberale Anstrich nicht hinwegtäuschen könne. Israelis sollten deshalb den Kontakt mit deutschen Institutionen meiden. ${ }^{5}$ Bis zur Ankunft der ersten Schilumimgüter in Israel galt der

1 HAARETZ (Tel Aviv) vom 7. 1. 1952.

2 Buber gegen direkte Verhandlungen vom 9.1.1952, CZA, S35/31.

3 Interview mit Buber am 1. 1. 1952, CZA, S35/31.

4 Pee HaAton vom 16. 1. 1952; MA'ARIv (Tel Aviv) vom 21. 6. 1953; Awjd (Düsseldorf) vom 24. 6. 1953 und 9. 10. 1953; HAMBURGER ECHO vom 25.6. 1953.

5 Dr. Zwi Rudi, Abteilung für internationale Institutionen im israelischen Außenministerium, an Dr. Josef Karai, unabhängige biologische Laboratorien, Kfar Malal, vom 16. 12. 1948, ISA, 5567/4065/G. 
totale Boykott Deutschlands in der israelischen Politik als unerschütterliches Leitmotiv. Auf die Dauer ließ er sich jedoch offensichtlich nicht aufrechterhalten. 1950 protestierten Knessetabgeordnete noch gegen die Einfuhr deutscher Druckerzeugnisse und forderten deren Beschlagnahmung. Die Antwort der Regierung, wonach die Behörden ermächtigt seien, jede ohne offizielle Erlaubnis eingeführte Ware zu konfiszieren, beruhigte die Gemüter wieder. ${ }^{6}$ Jede Form von Kontakt zwischen israelischen und deutschen Regierungsämtern war verboten, außer in Schilumimangelegenheiten. ${ }^{7}$ Die Bewilligungspflicht für kulturelle Kontakte zwischen Israel und Deutschland war mindestens bis 1954 in Kraft. ${ }^{8}$

Erst der Wille mit der Bundesrepublik zusammenzuarbeiten, um die Errungenschaften des jüdischen Staates der Öffentlichkeit in Deutschland vorzustellen, bewirkte eine allmähliche Lockerung der streng antideutschen Haltung. Die Regierung habe nichts gegen die Veröffentlichung von Beiträgen über Israel und das Zeigen von Filmen in Deutschland, die das Leben in Israel porträtierten und es in einer positiven Weise darstellten, lautete die neue offizielle Formel ${ }^{9}$, der sich Regierungsvertreter in der Knesset und andere israelische Politiker und Beamte fortan häufig bedienten. Konkret ging es vor allem um die Aufführung israelischer Kinofilme und Opern in Deutschland. Vordergründig nahmen die Israelis nur eine Gelegenheit für Öffentlichkeitsarbeit wahr. In Wirklichkeit zeugte die neue Formel aber von einem neuen Verständnis von Deutschlands internationaler Rolle, begleitet von der Einsicht, daß Öffentlichkeitsarbeit in Deutschland bzw. die Pflege eines positiven Israelbildes in der deutschen Bevölkerung mit Blick auf die Zukunft unerläßlich sei. Der naive Standpunkt, „Deutschland und die Deutschen, ob gute oder schlechte, interessieren uns nicht", war nicht mehr haltbar. Die neue Formel bedeutete faktisch das Ende des Boykotts. Schon bald entwikkelten sich regelmäßige beiderseitige Kontakte, wie sie zwischen Gesellschaften, Völkern und Staaten üblich sind.

Das israelische Außenministerium war sich der Sonderstellung kultureller und anderer öffentlicher Beziehungen mit Deutschland bewußt und suchte nach geeigneten Formeln. ${ }^{10}$ Das Thema Deutschland war so heikel, daß selbst Detailfragen in diesem Zusammenhang auf Regierungsebene beraten wurden. So kam die Frage der Vorführung des deutschsprachigen Kinofilms "Der Prozeß" nach dem gleichnamigen Roman von Franz Kafka in Israel zweimal im israelischen Kabinett zur Sprache und stellte die Minister vor eine schwierige Entscheidung: Kafka, einer der bedeutendsten jüdischen Schriftsteller des 20. Jahrhunderts, hatte seine Werke in deutscher Sprache geschrieben. Die Mehrheit stimmte schließlich für die Vorführung des Films. ${ }^{11}$ Im August 1955 ermächtigte die Regierung den Minister-

6 KNESSET-PROTOKOLLE [hebr.], 136. Sitzung am 8. 10. 1950, S. 22.

7 Finanzministerium an das israelische Außenministerium vom 15. 11. 1954, ISA, 2539/3a.

8 Direktor der Westeuropaabteilung an das Ministerbüro vom 10. 5. 1954, ISA, 3099/25.

9 Direktor der Westeuropaabteilung an das Ministerbüro vom 6. 10. 1954, ISA, 2413/3.

10 Yachil an Shinnar vom 7. 2. 1952, ISA, 613/7.

11 Protokoll der Kabinettssitzung Nr. 31/315 vom 20. 2. 1955 and Nr. 32/315 vom 27. 2. 1955, ISA, 7265/41. 
präsidenten und den Außenminister, über die Teilnahme Israels an internationalen Kongressen in Deutschland zu entscheiden. ${ }^{12}$

Anfang $1961 \mathrm{kam}$ es im Außenministerium zu einer Aussprache über das Thema israelische Kulturdarbietungen in Deutschland. Hierin beklagte sich der Direktor der Westeuropaabteilung beim Generaldirektor über die geringe Zahl solcher Darbietungen in der Bundesrepublik. Später kritisierte er schriftlich auch das gänzliche Ausbleiben deutscher Kulturauftritte in Israel. ${ }^{13}$ Statt wie zuvor kulturelle Kontakte zwischen Israel und der Bundesrepublik zu verhindern, ging das israelische Außenministerium nun dazu über, sie aktiv zu fördern. Doch die Schwierigkeiten waren noch lange nicht überwunden.

Ende 1960, kurz vor der Eröffnung des Eichmannprozesses, machte Dr. Scheiholz, ein deutscher Autor und persönlicher Freund von Bundespräsident Heinrich Lübke, den Vorschlag, während des Prozesses eine Delegation von israelischen Pädagogen in die Bundesrepublik einzuladen. ${ }^{14}$ Der ebenso gutgemeinte wie demonstrativ gemachte Vorschlag wurde in der Bundesrepublik vorwiegend positiv aufgenommen. Lübke förderte ihn mit viel Enthusiasmus, und niemand wagte es, Zweifel daran zu äußern. ${ }^{15}$ Schließlich begab sich eine vierköpfige israelische Pädagogendelegation auf Studienreise in verschiedene westeuropäische Länder, darunter auch die Bundesrepublik. Auf ihrem Programm stand der Besuch diverser Institutionen und die Begutachtung von Lehrmitteln für die technische Ausbildung. Die Unkosten des Aufenthalts in der Bundesrepublik wurden von den Gastgebern getragen. Obwohl der Schwerpunkt des Besuchprogramms im technischen Bereich lag, wurden den israelischen Pädagogen auch Geschichtsbücher und darin vor allem die dem Holocaust gewidmeten Kapitel gezeigt. ${ }^{16}$ Die Delegation hinterließ bei ihren Gastgebern einen glänzenden Eindruck. Als sich der Aufenthalt der israelischen Pädagogen in der Bundesrepublik dem Ende zuneigte, wurde die Sache publik und führte umgehend zu Protesten der israelischen Opposition. Darauf kam die Angelegenheit sowohl in der Knesset als auch im Kabinett zur Sprache.

Am 12. November 1961 beauftragte die Regierung Israels einen Regierungsausschuß mit der Ausarbeitung von Richtlinien für die Beziehungen mit Deutschland in den Bereichen Bildung und Kultur. ${ }^{17}$ Dieser empfahl die Einsetzung eines ministeriellen Steuerungsausschusses und eines Ministerausschusses für laufende Angelegenheiten. Der Steuerungsausschuß trat am 26. Dezember $1961 \mathrm{zu}$ einer ersten Sitzung zusammen, um die Richtlinien der Regierung zu beraten und einen Handlungsrahmen zu entwerfen. Er hatte den Auftrag, in sämtlichen ihm vorgelegten den Kulturaustausch mit Deutschland betreffenden Fragen zu entscheiden, konnte aber auch selbst die Initiative ergreifen. Internationale Veranstaltungen mit deutscher Beteiligung, wie zum Beispiel Fußballturniere, gehörten ebenfalls in

12 Ilsar an den Direktor der Westeuropaabteilung vom 29.6. 1956, ISA, 3309/25.

13 Direktor der Westeuropaabteilung an den Generaldirektor vom 18. 3. 1961, ISA, 3309/13.

14 Direktor der Westeuropaabteilung an Ministerin Meir vom 28. 6. 1961, ISA, 3309/14.

15 Savir an Shinnar vom 28. 3. 1962, ISA, 3309/14.

16 Varon an Savir vom 11. 5. 1961, ISA, 3309/14.

17 Bendor an die Mission in Köln vom 17. 11. 1961, ISA, 302/8. 
seinen Entscheidungsbereich. Die israelische Regierung war, wie bereits dargelegt, daran interessiert, die kulturellen Errungenschaften des jungen Staates einem breiteren Publikum in Deutschland vorzustellen, weniger aber an deutschen Darbietungen in Israel. ${ }^{18}$

In der erwähnten Knessetdebatte warfen die Opposition und einige Mitglieder der Koalition der Regierung mangelndes Einfühlungsvermögen sowie schrankenund gedankenlose Zusammenarbeit mit westdeutschen Institutionen vor. Die öffentliche Debatte drehte sich um das Versagen des deutschen Erziehungssystems und dessen Verknüpfung mit dem Nationalsozialismus und dem Dritten Reich. Das - im Vergleich zu anderen Äußerungen gemäßigte - Motto der Opposition lautete: „Vom deutschen Bildungswesen und von deutschen Pädagogen haben wir nichts zu lernen. " ${ }^{19}$ Die Kritik konzentrierte sich auf die Zusammenarbeit im geisteswissenschaftlichen Bereich, obwohl sich die Studienreise hauptsächlich auf die technische Ausbildung und Lernhilfen in diesem Bereich konzentrierte. Der Bildungsminister betonte vor der Knesset, wie wichtig es für Israel sei, die antinazistischen Tendenzen in der deutschen Bevölkerung zu stärken. Doch die Regierung war nicht interessiert an dieser Debatte, und Außenministerin Meir versuchte beim Knessetvorsitzenden deren Abbruch bzw. Verschiebung zu erwirken. ${ }^{20}$ Israelische Regierungskreise hielten die Kritik der Opposition für ungerechtfertigt, denn es fehle nicht an Mitteln der Überwachung der kulturellen Beziehungen zu Deutschland. Außerdem sei Israel an der Vertiefung der Beziehungen zu Deutschland interessiert. Die Einladung der israelischen Pädagogen wurde als Geste des guten Willens, eine unbeholfene Geste vielleicht, bewertet, der es angesichts des laufenden Eichmann-Prozesses an Fingerspitzengefühl gefehlt haben mochte, die jedoch offensichtlich gut gemeint gewesen sei. Der Antrag der Opposition auf Abbruch sämtlicher kultureller Kontakte mit der Bundesrepublik Deutschland wurde mit 37 zu 25 Stimmen abgelehnt.21

Statt dessen billigte die Knesset die von der Regierung ausgearbeiteten Vorschriften für den akademischen Austausch mit Deutschland. Diese sahen einschränkende Auflagen für israelische Studienaufenthalte in Deutschland vor, außer in Fächern, die in Israel nicht angeboten wurden. Weitere Beschränkungen galten sodann für den Austausch von Delegationen, gegenseitige Besuche von Vertretern kultureller und philanthropischer Organisationen sowie für die $\mathrm{Zu}-$ sammenarbeit von Institutionen im Hochschulbereich. Und schließlich versuchten die neuen Vorschriften, Ordnung in den Jugend- und Studentenaustausch zu bringen.22 Die westdeutsche Öffentlichkeit und besonders die Freunde Israels waren schockiert und entrüstet zugleich. Lübke gab der Befürchtung Ausdruck,

18 Protokoll der 1. Sitzung des Interministeriellen Ausschusses für kulturelle Beziehungen mit Deutschland vom 26. 12. 1961, ISA, 3309/15.

19 KNESSET-PROTOKOLle [hebr.], 38. Sitzung am 28. 10.1961, S. 494 und 61. Sitzung am 2. 1. 1962, S. 850-912; DEUTSCHKRON, Israel und die Deutschen, S. 187-189.

20 Bendor an Meroz und Varon an Shinnar vom 12. 10. 1961, ISA, 3309/15.

21 NACHRICHTENSPIEGEL vom 10.1. 1962; Rundschreiben der Israel-Mission vom 11. 1. 1962, PA, 708, 81.00-82.03, 92.19.

22 Westeuropaabteilung an die Mission in Köln vom 28. 12. 1961, ISA, 302/8; TIMEs (London) vom 28. 12. 1961. 
daß das mit viel Mühe Erreichte im kulturellen Bereich damit zunichte gemacht werde. ${ }^{23}$ Die Israel-Mission bedauerte den Schaden für das Ansehen des Staates Israel in der Bundesrepublik: „Wir werden hier in Köln die in Jerusalem eingeworfenen Scheiben reparieren müssen", hieß es. ${ }^{24}$

Doch die neuen Vorschriften waren nicht so einschneidend, wie zunächst befürchtet. In Wirklichkeit wurde dadurch in erster Linie Ordnung in ein Gebiet gebracht, wo vorher Willkür geherrscht hatte. Die Vorschriften zogen einerseits einen Schlußstrich unter den Kulturboykott und stellten andererseits endgültig klar, was erlaubt und was verboten war. Sie eröffneten zugleich neue Möglichkeiten für den Künstleraustausch, und für strittige Fragen gab es nun eine Klärungsstelle. Zudem entzogen sie den Kritikern die Grundlage. Die neuen israelischen Vorschriften für den Kulturaustausch mit Deutschland leiteten eine neue Phase in den deutsch-israelischen Kulturbeziehungen ein.

\section{Bücher, Bibliotheken und Archive}

Bereits in frühen Jahren entbrannte in jüdischen Kreisen ein Streit um die Verwendung der deutschen Sprache, die bei so manchem Holocaust-Überlebenden Erinnerungen des Grauens weckte und den Zuhörer leicht in Panik versetzen konnte. Die Opposition gegen die Verwendung der deutschen Sprache beruhte nicht auf rationalen Argumenten. Hinweise auf die großen Errungenschaften der deutschen Kultur und der deutsch-jüdischen Zivilisation, oder darauf, daß die Sprache ein neutrales Instrument der Zivilisation sei, fanden kein Gehör. ${ }^{25}$ Das Deutsche war in den Augen dieser Gruppe in erster Linie die Sprache des brüllenden SS-Mannes. Etwas komplizierter war die Situation in Israel, wo eine große Einwanderergemeinde aus Deutschland lebte, die das Deutsche als ihre Muttersprache betrachtete. Eine der Gründe für die in Israel verbreitete Abneigung gegen die sogenannten Jecken (aus Deutschland eingewanderte Juden) war der Umstand, daß sie untereinander in der verhaßten Sprache kommunizierten, wenn es auch unter Einwanderern aus Deutschland solche gab, für die das Deutsche den Nationalsozialismus versinnbildlichte. Auf dem Boden dieser emotionalen Abneigung konnten die ideologischen Strukturen der Deutschfeindlichkeit gedeihen, die von Politikern, Patrioten und Populisten für demagogische Zwecke mißbraucht wurden.

1952 erreichte der Streit über die deutsche Sprache mit der Hebräischen Universität in Jerusalem zum ersten Mal die Hochschulebene. Bibelforscher legten dar, daß die Kenntnis der deutschen Sprache für das Verständnis der Arbeiten der deutschen Bibelforschung unerläßlich sei, und forderten die Universitätsleitung auf, den Deutschunterricht einzuführen. ${ }^{26}$ Sprachwissenschaftler, Historiker und

23 Savir an Shinnar vom Februar 1962, ISA, 302/8.

24 Tavor an Shinnar vom 5. 2.1962, ISA, 302/8.

25 Das Motto „Ein Volk von Dichtern und Denkern“ wurde in „Ein Volk von Dichtern, Denkern und Henkern" abgeändert.

26 Zur Debatte über die Einführung der deutschen Sprache an der Hebräischen Universität: 
andere Gelehrte unterstützten diese Forderung. Dazu zählte der Philosoph Shmuel Hugo Bergmann, ein enger Freund Bubers, der versuchte, die Öffentlichkeit von der Notwendigkeit dieser Sprache zu überzeugen. ${ }^{27}$ Hiergegen opponierte der bekannte hebräische Dichter David Shimoni und setzte sich zunächst auch durch. Zwar sprach sich eine Mehrheit in den an der deutschen Sprache interessierten Fakultäten für die Einführung des Deutschunterrichts aus. Im Exekutivkomitee der Universitätsleitung überwogen jedoch die Neinstimmen, so daß der Deutschunterricht erst in den späten fünfziger Jahren legal in den Studienplan eingefügt wurde.

Auch Bücher in deutscher Sprache gerieten in Gefahr auf den Index gesetzt zu werden. Israel verfügte in jenen Jahren über ein relativ großes deutschsprachiges Lesepublikum. Zahlreiche israelische Akademiker hatten ihre Studien an deutschen Universitäten absolviert und pflegten regelmäßigen Kontakt mit deutschen Kollegen. Selbst der Nationalsozialismus und seine Unkultur taten dem Interesse an der deutschen Kultur und an den Errungenschaften der deutschen Universitäten bei diesen keinen Abbruch. Gegner des Nationalsozialismus und kulturelle Kreise in Deutschland erkannten das Potential des Buches als Botschafter des guten Willens und schickten Bücher an Freunde und Bibliotheken in Israel. Diese erreichten ihre Adressaten anfangs aber nur zum Teil. Denn mittels Sonderbestimmungen wurde die Einfuhr von in Deutschland gedruckter Lektüre eingeschränkt, und dem Einfuhrverbot fielen selbst ins Deutsche übersetzte und in Deutschland verlegte amerikanische Publikationen wie etwa die Zeitschrift Reader's Digest ${ }^{28}$ zum Opfer. Knessetabgeordnete protestieren gegen die Zuteilung von Fremdwährung für die Einfuhr deutscher Presseerzeugnisse, eine verwaltungsmäßige Absurdität, mit der sich die israelische Bildungselite nicht abfinden wollte. Wegen der strengen Devisenbeschränkungen sahen sich akademische Institutionen wie die Nationalbibliothek und der israelische Wissenschaftsrat (die spätere Israelische Akademie der Wissenschaften), gezwungen, Vertreter der Jewish Agency for Palestine (JAFP) in Deutschland zu bitten, wissenschaftliche Literatur für sie einzukaufen und ihnen mit der Post zuzuschicken. ${ }^{29}$ Schließlich erhielt die Nationalbibliothek vom Deutschen Verleger- und Buchhändlerverband eine jährliche Spende von hundert Büchern. Auch die jüdische Gemeinde in Mainz erklärte sich bereit, die israelische Nationalbibliothek unentgeltlich mit deutschen Büchern zu versorgen. Weitere Bücherspender kamen hinzu. ${ }^{30}$

KnesSET-Protokolle [Original hebr.], 4. Knesset, Sitzungen am 6. 4. 1960 und 3. 8. 1960; Dawar (Tel Aviv) vom 24. 10. 1952 und 6. 12. 1952; MaARIV (Tel Aviv) vom 12.8. 1953.

27 Dawar (Tel Aviv) vom 17., 24. 10. und 6. 12. 1952.

28 HaAretz (Tel Aviv) vom 12. 2. 1950.

29 Woermann an Landauer vom 8. 6. 1950; Landauer an Eschkol vom 4. 5. 1953, CZA, S35/ 163; Das Israelische Wissenschaftskomitee an Landauer vom 8.6. 1950, Kreutzberger an Gerling vom 4. 12. 1950, CZA, S35/162.

30 Erinnerung von Dr. Woermann vom 14. 7. 1952, CZA, S 35/88; Yachil an Woermann vom 19. 1. 1954, ISA, 617/16; Vermerk vom 23. 8. 1960, PA, 708, 82.60, 92.19; Vermerk Ref. 708, Ruyter, betr. Neue Film Verleih GmbH vom 23. 8. 1960, PA, 708, 82.60, 92.19; Nr. 1035; Dr. Rowas, Ref. 605, an die Film Presse Agentur in München vom 22. 4. 1960, PA, 708, 82.60, 92.19. 
Die Mission in Köln veranlaßte die Übersetzung hebräischer Werke ins Deutsche und umgekehrt, half bei der Ausfuhr deutscher Bücher nach Israel und gab entscheidende Impulse für die Gründung der Anne Frank-Stipendienstiftung für israelische Studenten des Fischer Verlages, Herausgeber der Tagebuchaufzeichnungen von Anne Frank in deutscher Sprache. Das Auswärtige Amt beschäftigte sich seinerseits mit der Herausgabe und Verbreitung von Büchern über Israel aufgrund aktueller politischer Ereignisse, etwa während des Eichmannprozesses. ${ }^{31}$ Der Staat Israel unterstützte die Veröffentlichung von propagandistischer Literatur über Israel in Deutschland, einschließlich solcher von Kirchenverlagen. Deutsche Schulbuchverlage baten um die Zusammenarbeit mit israelischen Historikern, besonders zum Thema Holocaust. ${ }^{32}$ Und die Stadtbücherei Mainz vergab ein Stipendium für Bibliothekskunde an einen israelischen Studenten, dessen Deutschlandaufenthalt vom interministeriellen israelischen Ausschuß für die Auswahl von Kandidaten für Stipendienprogramme gutgeheißen wurde. ${ }^{33}$

Israelische Gelehrte, allen voran Gershom Scholem, bemühten sich um die Rettung jüdischer Archive, die den Holocaust überdauert hatten, und forderten deren Überführung nach Israel oder wenigstens die Aufnahme auf Mikrofilm. Ein bekanntes Beispiel ist das Archiv der jüdischen Gemeinde Worms, das im Gegensatz zum Inhalt des berühmten Raschi-Hauses dank Adenauers persönlicher Intervention vollständig nach Israel transferiert werden konnte. ${ }^{34}$ Auf ähnliche Weise gelangten Archive kleinerer jüdischer Gemeinden in Bayern nach Israel. Das Archiv der jüdischen Gemeinde Hamburg wurde dagegen auf Mikrofilm aufgenommen, nachdem sich die Stadt Hamburg und die lokalen Hochschulbehörden geweigert hatten, es dem jüdischen Staat zu überlassen. ${ }^{35}$ Scholem unternahm zudem große Anstrengungen für die Überführung antiker jüdischer Bücher aus deutschen Bibliotheken nach Israel, quasi als Ersatz für die von den Nationalsozialisten zerstörten jüdischen Kulturschätze.

Interessiert zeigte sich Israel auch an der Übernahme des Archivs des Internationalen Suchdienstes in Arolsen. Das Archiv des Suchdienstes, der zur Auffindung und Identifizierung von Personen, deren Spur sich im Verlauf des Krieges verloren hatte - darunter Verschleppte, Exilanten, Konzentrationslagerhäftlinge und Zwangsarbeiter -, eingerichtet worden war, enthielt Informationen über rund eine Million Personen, ein Drittel davon Juden. Israel (und jüdische Organisationen) zählten zu den neun Staaten, die sich am meisten für die Erhaltung dieser Datensammlung einsetzten. Als sich 1953 das Ende des Besatzungsregimes abzeichnete, stellte sich die Frage, was mit dem Archiv des Suchdienstes geschehen sollte. ${ }^{36}$ Gemäß Deutschlandvertrag verpflichtete sich die Bundesrepublik, die

31 Verlag Heinz P. Conte Sprendlinger an den Informationsdienst Ausland des AA vom 5. 8. 1960; Ref. 993 an Ref. 708 vom 17. 8. 1960, PA, 708, 82.60, 92.19.

32 DIE WeLT (Hamburg) vom 25. 8. 1960.

33 Wörmann an Yachil vom 22. 12. 1961, ISA, 3309/15.

34 Shinnar an Ilsar vom 29.10.1956, ISA.

35 ISA, 613/16 (ganze Akte).

36 Bericht von Dr. Chaim Yachil betr. Internationaler Suchdienst vom 21. 12. 1953, ISA, 411/ $<$ 
bisherigen Tätigkeiten des Suchdienstes weiter zu unterstützen. ${ }^{37}$ Doch die israelische Regierung war nicht glücklich über diese Lösung, da die Archive in den Verantwortungsbereich von Bundesminister Theodor Oberländer, eine der umstrittensten Figuren in Adenauers Kabinett, gefallen wären. Der Bundesminister für Vertriebene, Flüchtlinge und Kriegsgeschädigte wurde beschuldigt, an der Vernichtung von Juden teilgenommen zu haben und erschien deshalb nicht der geeignete Mann für die Aufsicht über ein solches Archiv. ${ }^{38}$ Nicht nur die Israelis befürchteten, die Deutschen könnten Teile der Datenbank verfälschen, um die Identifizierung von Kriegsverbrechern zu verhindern. Das Archiv enthielt auch wertvolle Information für Entschädigungsberechtigte. Die Vereinigten Staaten befürchteten, Teile des Archivs könnten in die Sowjetunion überführt und anschließend gegen eigene Bürger verwendet werden. Israel forderte, die Daten des Archivs vollständig auf Mikrofilm aufzunehmen, um eine Kopie im HolocaustGedenk- und Forschungsinstitut Yad Vashem aufzubewahren. Diese Forderung wurde akzeptiert. Das Archiv blieb schließlich in Arolsen und wurde unter die Verantwortung des Roten Kreuzes gestellt. Die Leitung der Sammlung übernahm ein internationaler Ausschuß.

\section{Film, Theater und Musik}

Am 28. Oktober 1950 untersagte die dem Innenministerium unterstellte israelische Film- und Theaterzensur die Aufführung deutschsprachiger Filme und Theaterstücke in Israel. ${ }^{39}$ Diese Entscheidung war der Anfang einer jahrzehntelangen Debatte über die Angemessenheit der deutschen Sprache in öffentlichen Darbietungen in Israel. Neben Theater und Film betraf das Verbot auch Vokalmusik, öffentliche Lesungen und Vorlesungen, mit anderen Worten, fast jede Verwendung der deutschen Sprache im öffentlichen Rahmen. Zudem wurde Deutsch als Alltagssprache der deutschsprachigen Bevölkerungsteile diskriminiert und die Einwanderer aus Deutschland verschiedenen „ethnisch“ bedingten Schikanen ausgesetzt. Was die „Ostjuden “ in Deutschland durch die deutschen Juden hatten über sich ergehen lassen müssen, zahlten sie den "Jecken" im Heiligen Land nun heim - zuerst unter dem Vorwand der Priorität des Hebräischen und später unter dem Deckmantel des „Widerstandes gegen die Nazisprache“. Deutsche Lesungen mußten im Untergrund stattfinden. ${ }^{40}$ Deutschsprachigen Autoren standen schwere Zeiten bevor. Der Bann über die deutsche Sprache ist also nicht nur als innere Auflehnung gegen den Nationalsozialismus zu werten, sondern auch als Teil des jahrzehntelangen Ringens der zionistischen Bewegung um die Vormachtstellung des Hebräischen und als eine Art „sozioethnischer" Intoleranz gegenüber dem deutschen Judentum.

37 Ebd.

38 Memorandum des Internationalen Suchdienstes vom 24. 12. 1954; Abteilung für die Vereinten Nationen an Abraham Harman, New York, vom 22. 2. 1955, ISA, 411/6.

39 MA'ARIv (Tel Aviv) vom 29.11. 1950.

40 Vorlesungen von Albert Bassermann vom Dezember 1951, ISA, 532/10. 
Der Boykott der deutschen Sprache beschränkte sich nicht nur auf das Inland. Die Teilnahme der bekannten israelischen - aus Deutschland stammenden Schauspielerin Orna Porath an einer öffentlichen Lesung in Berlin auf Einladung der Israel-Mission führte umgehend zu Protesten in Israel. 41 Ähnliche Reaktionen rief eine Koproduktion des israelischen Staatsradios mit Radio Hamburg über Israel hervor. ${ }^{42} \mathrm{Die}$ israelische Regierung hatte offiziell nichts gegen israelische Darbietungen in Deutschland einzuwenden, und auch das Auswärtige Amt in Bonn gab sich wohlwollend, obwohl man arabische Proteste befürchtete. In der Antwort auf eine Anfrage bezüglich der Einladung des Jüdischen Theaters aus Warschau nach Deutschland wollte Voigt wissen, warum man denn ausgerechnet ein jüdisches Theater aus Polen und nicht direkt aus Israel eingeladen habe und fügte hinzu: „Hiervon abgesehen wären vom Standpunkt unserer Beziehungen zu Israel keine Bedenken zu erheben. ${ }^{43}$ Tatsächlich erschien noch im selben Jahr eine israelische Theatergruppe auf deutschen Bühnen. Ihre Auftritte mußten jedoch aufgrund des dürftigen Niveaus der Schauspieler kurzfristig wieder abgesagt werden. ${ }^{44}$

Der intensivste Bereich des deutsch-israelischen Kulturaustauschs war der Film. Hierzu wird das Zeigen israelischer Filme in Deutschland und umgekehrt sowie die Produktion von Filmen über Israel in Deutschland für das deutsche Publikum gezählt. Deutsche Filmemacher produzierten proisraelische, an Propaganda grenzende Filme für ein neugieriges deutsches Publikum, zum Teil mit staatlicher Unterstützung aus Bonn und Jerusalem. Bereits am 27. Januar 1953, also noch vor der Abstimmung über das Schilumimabkommen im Bundestag, prüfte das Auswärtige Amt Möglichkeiten der Filmproduktion in Israel. ${ }^{45}$ Etwa zur selben Zeit kam es zu ersten Interpellationen in der Knesset bezüglich der Vorführung israelischer und auf israelische Anregung produzierter Filme in Deutschland. Die offizielle Stellungnahme der Regierung, die längere Zeit beibehalten wurde, lautete: Israel sieht keinen Grund, in Deutschland keine Filme zu zeigen, die das Leben in Israel beschreiben. ${ }^{46}$

Eine deutsch-israelische ${ }_{n}$ Filmbegegnung ${ }$ anderer Art war die illegale Israelreise des jungen deutschen Pionierfilmers Thomas Harlan. Harlan wollte, wie er erklärte, quasi die Sünden seines Vaters, des berüchtigten NS-Filmregisseurs Veit Harlan, wiedergutmachen. Sein plötzliches Auftauchen in Israel führte zu tumultartigen Szenen, die das Eingreifen der Polizei erforderten. ${ }^{47}$ Harlans Werk veranlaßte ein Knessetmitglied der rechtsgerichteten Cherut zu heftiger Kritik an der Regierung. Der deutsche Journalist Rolf Vogel produzierte in Israel zwei Filme und rief damit den Protest arabischer Diplomaten in Bonn hervor, der das Aus-

41 KNESSET-Protokolle [hebr.], 458. Sitzung am 20. 7. 1954, S. 2189; Mn'ARIV (Tel Aviv) vom 4. 7. und 9. 8. 1954 .

42 KNESSET-PROTOKOLLE [hebr.], 435. Sitzung am 18. 1. 1965, S. 940.

43 Ref. 708 an das Ref. 605 vom 2. 11. 1960 und vom 24. 2. 1961, PA, 1035, 708, 82.60, 92.19.

44 Vermerk betr. israelisches Gastspiel vom 2. 11. 1960, PA, 708, 82.60, 92.19.

45 Henske, AA Berlin, an das AA, Bonn, vom 27. 1. 1953 und vom 2. 2. 1953, PA, 244-13 II.

46 Ilsar an den Direktor der Westeuropaabteilung vom 2. 6. 1953, ISA, 3309/25.

47 MA'ARIV (Tel Aviv) vom 19.6.1953. 
wärtige Amt dazu veranlaßte, die Filme zu prüfen. ${ }^{48}$ Die Bundesregierung beteiligte sich an der Produktion eines Films über die Kriegsverbrecherjustiz. 1958 wurde ein weiterer Dokumentarfilm über Israel gedreht. Eine Intervention des Auswärtigen Amts verhinderte dagegen die deutsch-israelische Koproduktion

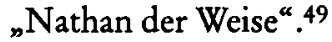

Israel exportierte mehrere Spielfilme in die Bundesrepublik, wahrscheinlich alle ohne jeden künstlerischen Wert. Im israelischen Außenministerium war man sich des propagandistischen Werts der Vorführung dieser Filme, insbesondere auch als Gegengewicht zur arabischen Propaganda, bewußt. ${ }^{50}$ Doch dem freien Vertrieb israelischer Filme in Deutschland waren Grenzen gesetzt. Einen Vorschlag von Goldmann vom Februar 1955, den international angesehenen Film „The Hill 22 Does Not Respond “ auch in Deutschland zu zeigen, beantwortete Shinnar mit dem Hinweis auf die in der Filmbranche übliche - im Falle Israels offensichtlich problematische - Gegenseitigkeit. ${ }^{51}$ Der erste in Israel zugelassene deutschsprachige Spielfilm war eine österreichische Produktion und handelte von der Blutlegende von Tiszaeszlar, Ungarn, im 19. Jahrhundert. Die Vorführung der Kafkaverfilmung „Der Prozeß ${ }^{4}$ führte, wie bereits erwähnt, zu größeren Debatten. ${ }^{52}$

Die israelische Regierung mußte sich früher oder später mit der heiklen Frage befassen, was mit deutschsprachigen Filmen schweizerischer und österreichischer Produktion geschehen sollte: Gilt der Boykott der deutschen Sprache oder Deutschland als Land? Wenn sich das Verbot gegen deutsche Produktionen richtet, weshalb sind dann davon auch schweizerische oder österreichische Produktionen betroffen? Richtet sich das Verbot gegen die deutsche Sprache, bedeutet dies eine Diskriminierung befreundeter Staaten. Und was soll mit deutsch-österreichischen Koproduktionen geschehen? Das israelische Kabinett widmete sich diesen Fragen am 30. Dezember 1956 anläßlich der geplanten Vorführung des österreichischen Films „Mozarts Leben“ und beschloß, österreichische und schweizerische Filme in deutscher Sprache fortan zuzulassen, ohne jedoch ein endgültige Regelung zu treffen..$^{53}$ Proteste des ehrwürdigen Rabbiner Nurock in der Knesset gegen diese Entscheidung wies die Regierung entschlossen zurück. ${ }^{54}$

Als nächstes stand die Vorführung „unanfechtbarer" deutscher Filme zur Debatte. Filmimporteure protestierten gegen das Verbot von Filmen deutscher Produktion und drohten dem Staat mit Schadenersatzklagen wegen dadurch entstandener Verluste. 1958 setzte Ben Gurion die Frage auf die Tagesordnung des Kabinetts ${ }^{55}$, das jedoch keine Lösung fand und die Frage ein weiteres Mal vertagte.

48 Aufzeichnung von Frowein vom 14. 11. 1955; Vermerk des AA vom 21. 2. 1956, PA, 1035, $708,82.20-82-70,92.19$.

49 Vermerk vom 22. 4. 1960, PA, 1035, 708, 82.60, 92.19.

50 Yachil an den Generaldirektor vom 22. 7. 1953, ISA, 617/16.

51 Goldmann an Shinnar vom 28. 2. 1955; Shinnar an Goldmann vom 21. 3. 1955, CZA, Z6/ 1996.

52 Rowas, AA, an die Film Presse Agentur \& Co. vom 22. 4. 1960, PA, 708, 82.60, 92.19.

53 MA'ARIV (Tel Aviv) vom 31. 12. 1956.

54 KNESSET-PROTOKOLLE [hebr.], 224. Sitzung am 16. 1. 1957, S. 775-776.

55 Bar-Yehuda an Ben Gurion vom 4. 2. 1958; Ben Gurion an Bar-Yehuda vom 4. 2. 1958, BGA, Correspondence File, 1958. 
Nachdem auch der Status von österreichischen Produktionen nicht endgültig geregelt werden konnte, reichte die österreichische Regierung beim israelischen Außenministerium offiziellen Protest gegen die Diskriminierung bzw. den Boykott österreichischer Filme ein. Darauf reagierte das israelische Außenministerium mit einer Rechtfertigung des Boykotts deutsch-österreichischer Koproduktionen. ${ }^{56}$ Ein endgültiger Regierungsbeschluß über deutsche Filme ließ aber weiter auf sich warten. In der Zwischenzeit verstärkte sich sowohl der Druck der Filmimporteure gegen das Verbot als auch der öffentliche Protest gegen die Aufführung deutschsprachiger Filme in Israel, angeführt von vier Organisationen ehemaliger NS-Widerstandskämpfer und KZ-Häftlinge. ${ }^{57}$ Diese Organisationen forderten ein generelles Verbot für die öffentliche Aufführung deutschsprachiger Filme in Israel. Die Swastikawelle der frühen sechziger Jahre der Bundesrepublik verschärfte den Disput noch zusätzlich, und die Regierung wurde mehrfach aufgefordert, endgültig Klarheit zu schaffen. Auf deutscher Seite protestierten deutsche Produzenten gegen die Diskriminierung durch Israel und forderten eine Intervention des Auswärtigen Amts. Dort gab man zu bedenken, daß eine solche Intervention die Lage allenfalls noch verschlimmern könnte. Die Sache gehöre „zu zahlreichen Einzelfragen der deutsch-israelischen Beziehungen, in denen nur Zurückhaltung und Geduld auf deutscher Seite zu gewünschtem Erfolg führen könne“, bemerkte dazu das Referat 708.58

Die meisten Auseinandersetzungen und Konflikte ereigneten sich aus unerfindlichen Gründen im Bereich Musik. Bis heute umstritten ist etwa die Frage, ob man die Werke des erklärten Antisemiten Richard Wagner oder des NS-Kollaborateurs Richard Strauss spielen bzw. vom Mitläufer Herbert von Karajan dirigierte Musik hören dürfe. Ja, selbst die deutschen Chortexte zu Beethovens IX. Symphonie und Schuberts Lieder gaben zu manchen Diskussionen Anlaß. Der Begeisterung der Liebhaber dieser Werke stand die Holocaust-Erinnerung entgegen. Die israelischen Komponisten machten einen besonders militanten Eindruck. Da sie jeden Kontakt mit deutschen Kulturinstitutionen ablehnten, war eine Verständigung über die Aufführung oder Aufnahme israelischer Werke in Deutschland von vornherein ausgeschlossen. ${ }^{59}$ Später bewilligte die israelische Regierung offiziell israelische Musikdarbietungen in Deutschland, um die Leistungen des jungen Staates einem breiten Publikum vorzustellen. Ungeachtet der fortdauernden Boykottdebatte verschafften sich die israelischen Musikliebhaber sowohl Zugang zur modernen deutschen Musik als auch zu den Klassikern. 1965 erschienen in Israel zum ersten Mal Pressenotizen über die Einladung eines Berliner Orchesters, die jedoch umgehend dementiert wurden. Es könnte sich um einen Versuchsballon gehandelt haben.

56 Ref. 708 an Ref. 605 betr. Neue Film Verleih GmbH vom 23. 8. 1960, PA, 1035, 708, 82.60, 92.19.

57 DIE WELT (Hamburg) vom 6. 2. 1959.

58 Ref. 708 an das Ref. 606 vom 23. 8. 1960, PA, 1035, 708, 82.60, 92.19.

59 Das AA an die Israel-Mission vom 20. 10. 1953; Yachil an den Rechtsberater des israelischen Außenministeriums vom 11. 11. 1953, ISA, 617/16; AwJD vom 4. 6. 1954; Westeuropaabteilung an die Mission vom 2. 1. 1955, ISA, 610/22. 


\section{Sport}

Die im sportlichen Wettkampf unvermeidliche direkte Begegnung war für israelische Sportler, die mit deutschen Sportlern zusammentrafen, oft ein emotionsgeladenes Ereignis. Soweit es ging, mieden die Israelis Sportwettkämpfe mit deutschen Sportlern und Mannschaften. Zur ersten Begegnung im Sport zwischen einer deutschen und einer israelischen Mannschaft kam es anläßlich der Schacholympiade im Herbst 1952 in Stockholm. Die israelischen Schachspieler wurden angewiesen, der ägyptischen Mannschaft ohne Vorbehalte zu begegnen, den Deutschen gegenüber dagegen kühle Korrektheit zu demonstrieren. ${ }^{60}$ Bei der Schacholympiade von 1954 in Amsterdam spielte eine israelische gegen eine ostdeutsche Mannschaft, und im selben Jahr standen sich ein Israeli und ein Deutscher bei den Tennismeisterschaften in Wimbledon gegenüber. 1955 sprach sich das israelische Außenministerium gegen einen deutsch-israelischen Segelflugwettbewerb aus und vier Jahre später gegen ein Freundschaftsspiel der Fußballmannschaft RotWeiß Essen in Israel.61 Entsprechend riet die israelische Diplomatie einem israelischen Fußballspieler, eine Einladung der Mannschaft Victoria Köln abzulehnen. Im Vorfeld der Schachmeisterolympiade in Frankfurt an der Oder im Oktober 1960 schlug das israelische Außenministerium vor, den anderen westlichen Mannschaften zu folgen, d.h. wenn sie sich für eine Teilnahme entschieden, sollte es Israel auch tun. ${ }^{62}$ Der Kalte Krieg machte die Angelegenheit noch komplizierter: Frankfurt an der Oder lag nicht nur in Deutschland, sondern dazu noch im Osten. Insgesamt gab sich die israelische Regierung im sportlichen Bereich eher kompromißbereit. Offensichtlich hielt sie sich dabei an folgende Regel: Israel nimmt an internationalen Sportwettkämpfen mit deutscher Beteiligung teil, meidet aber deutsch-israelische Sportveranstaltungen.

\section{Wissenschaft, Ausstellungen, internationale Foren}

Das israelische Außenministerium riet israelischen Wissenschaftlern in der Regel von der Teilnahme an Konferenzen in Deutschland oder von der Annahme von Einladungen deutscher Institute ab. Sollte dennoch ein Besuch in Deutschland in Erwägung gezogen werden, so schlug das Außenministerium vor, zuerst die Kriegsvergangenheit der einladenden Institute oder Personen zu prüfen. Ein Wissenschaftler des Haifaer Technikon erhielt 1954 die Ehrendoktorwürde der Stuttgarter Universität, weigerte sich jedoch, den Titel dort in Empfang zu nehmen. Auf diesen Fall angesprochen, meinte Außenminister Sharett, der Wissenschaftler solle den Preis akzeptieren, sofern er ihn ohne eine Reise nach Stuttgart empfangen könne und eine gründliche Überprüfung des Instituts nicht gegen eine An-

60 Westeuropaabteilung an den Israelischen Schachverband vom 21. 7. 1952, ISA, 2539/2.

61 Ilsar an die Abteilung Vereinte Nationen vom 29. 7. 1955, ISA, 2539/4.

62 Shinnar an Savir vom 7. 3. 1960, ISA, 300/8. 
nahme spreche. ${ }^{63} \mathrm{Im}$ August desselben Jahres diskutierte das Kabinett die israelische Beteiligung an internationalen Zusammenkünften in Deutschland und übertrug die Entscheidung dem Ministerpräsidenten. ${ }^{64}$

Fast von Anfang an kauften israelische Institutionen in Deutschland nicht nur wissenschaftliche Literatur, sondern auch Geräte und Apparaturen. Israelische Forscher wurden dazu ermuntert, Beiträge in deutschen Fachzeitschriften zu veröffentlichen, nicht jedoch deutsche Beiträge in eigenen Zeitschriften zu akzeptieren. 1961 stellte der Generaldirektor des israelischen Gesundheitsministeriums fest, daß Beiträge israelischer Wissenschaftler in deutschen Fachzeitschriften erscheinen und daß deutsche Institutionen wissenschaftliches Material in Israel anfordern könnten, ohne den israelischen Medizinerverband zu konsultieren. ${ }^{65}$ Im wissenschaftlichen Verkehr zwischen beiden Ländern wurden schlicht Fakten geschaffen. Dabei unterstützte das Außenministerium die wissenschaftliche $\mathrm{Zu}$ sammenarbeit $\mathrm{zwischen}$ israelischen und deutschen Forschern, wie sich etwa in der Archäologie und der Meteorologie zeigte.66

Zwischen israelischen Forschern und dem Max-Planck-Institut der Heidelberger Universität entwickelten sich rege Kontakte auf verschiedenen Gebieten. Angeführt wurden diese Zusammenarbeit auf israelischer Seite vom WeizmannInstitut in Rehovot. Forscher des Max-Planck-Instituts und des Weizmann-Instituts trafen sich bereits 1956 außerhalb Israels zu Gesprächen über eine mögliche Zusammenarbeit zwischen beiden Instituten, die sich später zu einem intensiven wissenschaftlichen Austausch entwickeln sollte. ${ }^{67}$ Der Heidelberger Physiker Hans Jensen hielt 1957 im Weizmann-Institut die erste Vorlesung eines deutschen Wissenschaftlers in Israel. 1958 zahlte die Alexander von Humboldt-Stiftung zum ersten Mal einem israelischen Wissenschaftler - einer Rechtswissenschaftlerin einen Forschungszuschuß. 1959 lehrten am Weizmann-Institut zwei deutsche Kernphysiker, Wolfgang Gentner und Otto Hahn, als Gastdozenten. ${ }^{68} \mathrm{Ihr}$ Aufenthalt löste zahlreiche Gerüchte über eine deutsch-israelische Zusammenarbeit im Nuklearbereich aus. ${ }^{69}$ Obwohl die informelle - von der Regierung stillschweigend geduldete - Zusammenarbeit zwischen dem Weizmann-Institut und deutschen Wissenschaftlern später auch offiziell genehmigt wurde, kann doch behauptet werden, daß sich die Wissenschaftler in der Regel nicht um die Vorgaben der politischen Stellen kümmerten. Die amtlichen Boykottaufrufe und Sanktionen

${ }^{63}$ Der Direktor der Westeuropaabteilung an das Ministerbüro vom 10. 5. 1954, ISA, 3099/ 25.

64 Ilsar an den Generaldirektor vom 29. 6. 1954, ISA, 3309/25.

65 Dr. S. Suessmann, Generaldirektor das Gesundheitsministeriums, an Bendor, israelisches Außenministerium, vom 3. 12. 1961, ISA, 3309/15.

66 Das Büro des Generaldirektors an Shinnar vom 19. 2. 1955, ISA, 517/7; Ilsar an die Abteilung für die Vereinten Nationen vom 29. 7. 1955, ISA 2539/4.

67 Heatid (Tel Aviv) Nr. 32 von 1974.

68 Vermerk von v. Hase vom 28. 10. 1964, PA, B36, 110, 82.00, 92.19.

69 Zur Förderung des wissenschaftlichen Austauschs mit der deutschen Forschergemeinde durch das Weizmann-Institut: NiCKEL, Es begann in Rechovoth, S. 18-35; Vermerk von v. Hase vom 28. 10. 1964, PA, B36, 110, 82.00, 92.19; Vialon, Bundeskanzleramt, an Lahr, AA, vom 15. 11. 1961, PA 708, 82.60, 92.19. 
konnten israelische Wissenschaftler jedenfalls nicht davon abhalten, Kontakte mit deutschen Forschern und Forschungsinstituten zu knüpfen.

Anläßlich des 85. Geburtstags von Martin Buber bat die Ruhr-Universität Bochum um Erlaubnis, ihr Institut für Judaistik nach dem berühmten Philosophen benennen zu dürfen. ${ }^{70}$ Ein anderer Vorschlag betraf die Einrichtung eines Franz Oppenheimer-Lehrstuhls an der Hebräischen Universität, benannt nach dem bekannten deutsch-jüdischen Soziologen und Nationalökonomen, zu dessen Schülern auch Ludwig Erhard gezählt hatte. ${ }^{71}$

Die Aufgabe von israelischen Ausstellungen in der Bundesrepublik war es, die Neugier des westdeutschen Publikums über Israel zu befriedigen. Die erste israelische Ausstellung in Deutschland - „Alt-Neuland“ - fand 1954 statt. $^{72}$ Die israelischen Ausstellungen in Westdeutschland lassen sich in drei Hauptkategorien einteilen: Ausstellungen, die das Leben und die Landschaften Israels bzw. des Heiligen Landes beschrieben oder mit anderen Worten, zu Propaganda neigende PR-Ausstellungen für die breite Öffentlichkeit. Sie wurden in der Regel von der Israel-Mission angeregt. Eine weitere Kategorie betraf Fach- und Kunstausstellungen. Ausstellungen dieser Art beschäftigten sich mit israelischer Kunst, archäologischen Ausgrabungen, jüdischer Geschichte usw. und wurden entweder auf lokale Initiative, von Berufsverbänden oder ganz spezifischen Stellen in Israel durchgeführt. Israelische Kunstausstellungen kamen in manchen Fällen auf lokale Initiative, oft nicht aus künstlerischen Gründen, zustande. Die erste israelische Kunstausstellung in Deutschland wurde 1960 in Köln eröffnet. ${ }^{73}$ Schließlich sind die israelischen Handelsausstellungen zu erwähnen. Hierbei handelte es sich entweder um Einzelausstellungen, die Produkten der israelischen Landwirtschaft und Industrie gewidmet waren oder um Ausstellungen innerhalb allgemeiner deutscher Handelsmessen. Die israelischen Stände an solchen Messen, die sich in den fünfziger Jahren besonderer Beliebtheit erfreuten, zogen ein breites Publikum aus allen Bevölkerungsschichten sowie Schulklassen und Vereine von Freunden Israels an. Die erste bundesdeutsche Handelsmesse mit israelischer Beteiligung war die Frankfurter Messe von 1957. Fälle, in denen Einzelpersonen unberechtigt als Vertreter des Staates Israel auftraten und dem Ansehen des jüdischen Staates und seiner Ausfuhr dabei Schaden zufügten, veranlaßten die israelische Regierung, sich bei der Organisation von Ausstellungen im Ausland fortan stärker zu engagieren. ${ }^{74}$

Israelische Redner, besonders Persönlichkeiten wie Martin Buber und Max Brod sowie Diplomaten wie Yachil und Tavor, waren bei öffentlichen Anlässen, in Seminaren, Studentenzirkeln sowie in Intellektuellen- und Akademikerkreisen, an Hochschulen und in Kirchenvereinen sehr gefragt. Die antideutsche Lobby in der Knesset war nicht glücklich über den regen kulturellen Austausch, faktisch aber machtlos.

70 Gerstenmaier an Shinnar vom 17. 1. 1963, ISA, 322/8.

71 Vermerk des AA vom 20. 8. 64, PA, B36, 110, 82.00, 92.19.

72 AWJD vom 21. 5. 1954.

73 Deutsche Zeitung (Köln) vom 27. 9. 1960.

74 Savir an Shinnar vom 24. 5. 1959, ISA, 300/7. 


\section{Verkehrsverbindungen und Fernmeldeverkehr}

Der Fracht- und Personenverkehr zwischen deutschen und israelischen Häfen wurde im Jahre 1949 aufgenommen. ${ }^{75}$ Die ersten Frachter auf dieser Linie transportierten das persönliche Eigentum von DPs und wenig kommerzielle Fracht. Der angesichts des spärlichen Handels zunächst geringe Verkehr weitete sich später mit dem Beginn des Schilumimgüterflusses beträchtlich aus.

Auch der Post- und Telekommunikationsverkehr hatte anfänglich mit größeren Schwierigkeiten zu kämpfen. Am Anfang schickte die Deutsche Post die für Israel bestimmte Post zunächst nach Jordanien, wo sie oft verlorenging. Ein Abkommen über eine direkte Telephonverbindung wurde erst 1964 erzielt.

Die Herstellung einer regelmäßigen Flugverbindung dauerte etwa acht Jahre. Am 11. Juli 1951 stimmte das israelische Kabinett gegen direkte Flugverbindungen mit Deutschland 76 , eine Entscheidung, die sowohl im Ausland als auch in Israel selbst kritisiert wurde. Der politische Ausschuß der Mapai kommentiere sie mit der Bemerkung, man könne Deutschland nicht von der Landkarte streichen, und sprach sich für Flüge der staatlichen Fluggesellschaft El Al nach Deutschland aus. ${ }^{77} \mathrm{Am}$ nächsten Tag rollte das Kabinett die Frage wieder auf und beauftragte einen Ausschuß mit der Prüfung der Frage, ob El Al-Flugzeuge deutsche Flughäfen anfliegen sollten. ${ }^{78}$ Das israelische Außenministerium wies in diesem $\mathrm{Zu}$ sammenhang darauf hin, daß israelische Schiffe regelmäßig in deutsche Häfen einlaufen würden. Am 13. September 1953 landete zum ersten Mal ein israelisches (Fracht-)Flugzeug auf deutschem Territorium. ${ }^{79}$ Anfang 1954 reichte Shinnar sodann ein offizielles Gesuch für einen wöchentlichen El Al-Flug mit Fracht, Passagieren und Post nach Düsseldorf ein. ${ }^{80}$ Rund fünf Monate später, am 22. Juni 1954, landete dort der erste reguläre Flug aus Israel unter Ausschluß der Öffentlichkeit. Das Flugzeug hatte nur Fracht geladen und kehrte mit Schilumimgütern nach Israel zurück. Die erste El Al-Fluglinie nach Deutschland, via Rom nach Frankfurt am Main, wurde erst am 22. Februar 1958 eröffnet. Am ersten Flug nach Israel nahmen mehrere hohe Beamte des Auswärtigen Amts und des Bundeskanzleramts, jüdische Persönlichkeiten und Journalisten teil. ${ }^{81}$ Lufthansa folgte $\mathrm{El} \mathrm{Al}$ auf der Linie nach Tel Aviv. ${ }^{82}$ Nach der israelischen Schiffahrtsgesellschaft ZIM begann auch die Deutsche Levant Maritime Comp. auf der Linie Bremen-Haifa zu verkehren. Die regulären See- und Luftverbindungen bildeten die Grundlage für den zunehmenden Touristenverkehr zwischen beiden Ländern.

\footnotetext{
75 Dawar (Tel Aviv) vom 7. 3. 1949.

76 Protokoll der Kabinettssitzung Nr. 44/311 vom 11. 7. 1951, ISA, 7264/1.

77 Protokoll der Sitzung des politischen Komitees vom 28. 3. 1953, LPA, Protokolle des Politischen Komitees der Mapai.

78 Protokoll der Kabinettssitzung Nr. 28/313 vom 29. 3. 1953, ISA, 7264/9.

79 MA'ARIV (Tel Aviv) vom 13. 9. 1953.

80 Shinnar an die Alliierte Hohe Kommission vom 25. 1. 1954, ISA, 572/2b.

81 Coburger, Die Beziehungen, S. 189.

82 Ebd.
} 


\section{Der Reiseverkehr}

Der Reiseverkehr zwischen Eretz Israel und Deutschland nach 1945 durchlief verschiedene Phasen. Bei den ersten Reisen handelte es sich um Besuche von Bürgern des britischen Palästinamandats im besetzten Deutschland. Der Besucherstrom in Richtung Deutschland riß auch nach der Gründung des Staates Israel nicht ab, trotz behördlicher Schikanen. Demgegenüber war der von den israelischen Behörden stark behinderte Reiseverkehr in umgekehrter Richtung zunächst sehr spärlich. Einzelnen deutschen Staatsbürgern gelang die Einreise nach Israel dennoch. Später folgten Gruppen - Studenten und Jugendliche. Mit dem Aufkommen des israelischen Gruppenreiseverkehrs nach Deutschland bot sich dann die Möglichkeit von Austauschprogrammen. So wurde der gegenseitige Reiseverkehr vor allem zu Tourismus- und Pilgerzwecken - allmählich immer dichter.

Soldaten der jüdischen Palästinabrigade betraten als erste jüdische Bürger des britischen Palästinamandats deutsches Territorium. Später folgten verschiedene offizielle Vertreter, Sozialarbeiter, Lehrer usw. mit diversen Aufgaben im Zusammenhang mit den DPs. Schließlich folgten Kaufleute, Privatreisende, darunter Einzelpersonen mit Restitutionsansprüchen, Politiker und andere mehr. Die israelischen Behörden versuchten, die Ausreise in geordnete Bahnen zu lenken und die Zahl der israelischen Besucher in der Bundesrepublik zu begrenzen. Ein Ausdruck des Deutschlandboykotts war der Stempel in israelischen Pässen „not valid for Germany" (gilt nicht für Deutschland). Israelische Bürger, die einen Besuch in der Bundesrepublik planten, mußten im Innenministerium einen entsprechenden Antrag stellen. Die westdeutschen Behörden ignorierten den Stempel zunächst. Sie hatten keinen Grund, den israelischen Boykott zu unterstützen. So stellten, wie bereits erwähnt, westdeutsche Konsulate in Nachbarländern Sichtvermerke für Israelis aus und stempelten diese auf Anfrage auf ein Beiblatt anstatt direkt in den $\mathrm{Pa}$, um den Besucher vor strafrechtlicher Verfolgung zu schützen. ${ }^{83}$ Das britische Konsulat in Haifa, das israelische Konsulat in München und die kurzlebige israelische Vertretung in Berlin standen den israelischen Reisenden zu Diensten.

Als jedoch der israelische Tourismus in die Bundesrepublik anzuschwellen begann und ein Teil der Besucher beabsichtigte, sich in Deutschland niederzulassen, entdeckten die westdeutschen Behörden die Vorzüge des Stempels und benutzten ihn zur Ablehnung von Visumanträgen israelischer Bürger. Eine Zeit lang unternahmen die israelischen und westdeutschen Behörden gemeinsame Anstrengungen zur Begrenzung der Einreise von Israelis in die Bundesrepublik. Das Hauptmotiv auf israelischer Seite war längst nicht mehr der Deutschlandboykott. Vielmehr stand nun die zionistische Ideologie, die Eretz Israel als Heimat der Juden betrachtet, im Vordergrund. Deshalb wurde versucht, die dieser Ideologie widersprechende Auswanderung aus Israel zu verhindern. In der Bundesrepublik war das Verhalten der Behörden von antisemitischen Untertönen begleitet. Mit amtlichen Maßnahmen war man bestrebt, die Niederlassung von Juden in Deutsch-

${ }^{83}$ Shlomai an Shinnar vom 6. 8. 1953, ISA, 572/2a. 
land zu verhindern. 1958 wurden israelische Visumanträge ohne Begründung zurückgewiesen. ${ }^{84}$

Berichte der israelischen Tageszeitung Yediot Acbronoth über angebliche Diskrimierung israelischer Visumsanwärter in der Bundesrepublik dementierte Außenministerin Golda Meir in der Knesset ${ }^{85}$ und fügte hinzu, daß Regierungsvertreter auf Staatsreise, Kaufleute mit Bewilligung der Handelskammer, Akademiker und Lehrer mit offiziellem Auftrag sowie Entschädigungsanwärter ein einwöchiges Transitvisa erhielten und kein spezielles Visum bräuchten. Meirs Erläuterungen scheinen den Verdacht der deutsch-israelischen Zusammenarbeit in diesem Bereich endgültig zu bestätigen. Westdeutsche Stellen hatten zwar bereits 1955 erwogen, die Visumpflicht für israelische Besucher aufzuheben. Der Plan wurde jedoch aufgrund von Befürchtungen des Bundesinnenministeriums zurückgestellt, Israel könne sich weigern, unerwünschte Einreisende wieder aufzunehmen. ${ }^{86}$ Ein weiteres Hindernis war die ausschließlich von israelischen Besuchern geforderte Kaution von DM 1000. Der Sprecher der Bundesregierung bezeichnete die Kautionspflicht für israelische Bürger am 16. März 1960 im Bundestag als Vorsichtsmaßnahme, die verhindern sollte, daß Israelis in Deutschland illegal arbeiteten. Darauf wollte der SPD-Abgeordnete Adolf Arndt wissen, worin sich israelische Schwarzarbeiter denn von ihren italienischen und spanischen Kollegen unterschieden. ${ }^{87}$

Die israelische Regierung protestierte weder gegen diesen noch gegen andere Fälle von Diskriminierung. Sie wurde angesichts der Diskriminierung deutscher Visumanwärter in Israel und wegen der eigenen Absicht, den Aufenthalt von Israelis in Deutschland zu verhindern, stillschweigend in Kauf genommen. Der Stempel wurde am 1. Mai $1956 \mathrm{zwar}$ abgeschafft ${ }^{88}$, die Bewilligungspflicht für Deutschlandreisen damit jedoch nicht aufgehoben. Leitende Beamte des israelischen Außenministeriums erkannten aber schließlich, daß der Boykott gegen Deutschland nicht mehr zeitgemäß war, und legten 1961 Vorschläge für drastische Änderungen der Deutschlandpolitik vor. Nach dem Eichmann-Prozeß wurden die Beschränkungen für Reisen nach Deutschland aufgehoben. ${ }^{89}$

Der israelische Reiseverkehr nach Deutschland - Einzelreisende, Gruppen (darunter auch Soldaten) und Neueinwanderer, die ihre alte Heimat besuchten oder dorthin zurückkehrten - nahm stetig zu. Bei letzteren erfreute sich die doppelte Staatsbürgerschaft zunehmender Beliebtheit. Viele Israelis deutscher Herkunft sicherten sich neben der israelischen auch die deutsche Staatsbürgerschaft. Man kann davon ausgehen, daß die große Verbreitung von bundesdeutschen Pässen in Israel zur Abschaffung des erwähnten Stempels beigetragen hat. Angesichts

${ }^{84}$ DIE WeLT (Hamburg) vom 15. 7. 1958.

85 KNESSET-PROTOKOLLE [Original hebr.], 42. Sitzung am 1.2. 1960, S. 513.

86 Abt. Westeuropa an Shinnar vom 10. 1. 1955, ISA, 613/7; Naor an die Abt. Westeuropa vom 27. 1. 1955, ISA, 2588/23.

87 Artikel "Juden unerwünscht?" von Dr. Adolf Arndt im SPD-Pressedienst vom 19. 3. 1960, AdsD, SPD-Bundestagsfraktion, Nr. 468.

88 Scheck an Sharett vom 25. 4. 1956, ISA, 2539/4.

89 Der Direktor der Westeuropaabteilung an den Generaldirektor vom 18. 3. 1961, ISA, 3309/13. 
der verbreiteten Deutschfeindlichkeit ist die außergewöhnliche Dynamik des israelischen Erholungs- und Besichtigungstourismus in Deutschland mehr als erstaunlich. Andererseits ist daran zu erinnern, daß viele Israelis Deutschland bis heute boykottieren.

Der deutsche Reiseverkehr nach Israel entwickelte sich langsamer und war wiederholt Gegenstand interner Debatten und Konsultationen. Bei den ersten Bürgern der Bundesrepublik, die - 1950 - offiziell nach Israel eingeladen wurden, handelte es sich, wie erwähnt, um sogenannte Gerechte der Nationen wie etwa Kreisdekan Maas. Die ersten deutschen Journalisten, denen die israelischen Behörden die Einreise erlaubten, waren sodann bekannte Sympathisanten des jüdischen Staates, darunter Rudolf Küstermeier, Rolf Vogel und Erich Lüth. Alle drei mußten ihre Identität bei ihrem ersten Besuch aus Sicherheitsgründen verheimlichen, aber auch, um Skandale zu vermeiden. Prälat Meinartz aus Köln war der erste Vertreter der katholischen Kirche, der eine offizielle Einreiseerlaubnis erhielt. Er wurde von ein paar Pilgern begleitet, die sich Reisegruppen aus anderen Ländern anschlossen, und von Privatreisenden auf Verwandtenbesuch. ${ }^{90} \mathrm{Der} \mathrm{Be}$ sucherstrom sowohl von katholischen als auch von evangelischen Geistlichen aus Deutschland nahm ständig zu. Der erste deutsche Politiker, der israelisches Territorium betrat, war Franz Böhm. Josephthal machte im März 1953 den Vorschlag, Böhm nach Israel einzuladen, doch Ben Gurion lehnte dies mit Hinweis auf den großen Sicherheitsaufwand ab, den ein solcher Besuch erfordern würde. ${ }^{11}$ Doch bereits zwei Monate später, nach der Ratifizierung des Schilumimabkommens, erfolgte die Einladung.92

Der Besucherstrom aus Deutschland, gleichgültig in welchem Umfang, war der rechten Opposition ein Dorn im Auge. Auf eine parlamentarische Anfrage sagte Sharett am 29. Juli 1953 in der Knesset, daß bis zu jenem Zeitpunkt acht deutsche Staatsangehörige nach Israel eingereist seien, darunter drei "Gerechte der Nationen" und fünf Rentnerinnen auf Verwandtenbesuch. Etwa dreißig Gesuche seien in Behandlung, wobei sich das Außenministerium strikt an die bestehende Politik halte. ${ }^{93}$ Aus Sharetts Angaben könnte man schließen, daß der Staat Israel nur „Gerechte der Nationen" und nahe Verwandte von Einwohnern Israels einreisen ließ. Doch aus den Dokumenten geht hervor, daß zu den deutschen Besuchern in Israel längst auch proisraelische Journalisten und Politiker, Kirchenvertreter und Pilger zählten. Die Zulassungskriterien waren offensichtlich breiter gefaßt, als von Sharett zugegeben, und weiteten sich ständig weiter aus. ${ }^{94} 1954$ reiste ein deutscher Arzt in Israel ein, um Opfer der NS-Verfolgung zu untersuchen.

90 Schwartz an Landauer vom 18. 10. 1950, ISA, CZA, L47/148/1; Livneh an die Abt. Westeuropa vom 18. 10. 1951, ISA, 2539/2; Livneh an die Abt. Westeuropa vom 11. 10. 1951. ISA, 2539/1; LÜTH, Die Friedensbitte.

91 Tagebucheintrag Ben Gurions vom 22. 3. 1953, BGA, BGD.

92 Shinnar an Böhm vom 11. 5. 1953; Böhm an Shinnar vom 20. 5. 1953, CZA, Z6/1999.

93 KNesSET-Protokolle [Original hebr.], 3. Knesset, 279. Sitzung am 29. 7. 1953, S. 2065.

94 KNeSSET-Protokolle [Original hebr.], 3. Knesset, Sitzungen am 6. 3. 1954, S. 1199 und am 17. 3. 1954, S. 1219; Najar, Abt. Westeuropa, an Sahar, Israelischer Polizeichef, vom 16. 5. 1954 und 23. 5. 1954; Die israelische Polizei an den Außenminister vom 22. 5. 1954, ISA, 2546/11. 
Der zunehmende Strom von Visumsanträgen und Besuchern erforderte die Formulierung entsprechender Vorschriften. Das Außenministerium informierte die Grenzpolizei über die Voraussetzungen für die Einreise deutscher Staatsbürger wie folgt: Einreisen dürfe, wer keine NS-Vergangenheit habe, deutscher Staatsbürger jüdischer Abstammung oder Verwandter von israelischen Staatsbürgern sei. Einreisevisa erhielten zudem Geistliche mit Antinazivergangenheit, Pilger (sofern sie mit Gruppen aus anderen Ländern reisten), Konferenzteilnehmer, Spezialisten auf Einladung der Schilumimgesellschaft und Gäste des Außenministeriums. Die Polizei sprach sich gegen die Einreiseerlaubnis für nichtjüdische Verwandte von israelischen Bürgern aus, da man, wie es hieß, nicht für ihre Sicherheit garantieren könne. Das Außenministerium billigte diesen Einwand im Grundsatz und wies darauf hin, daß deutsche Besucher überall dort ein Sicherheitsrisiko darstellten, wo sie die Aufmerksamkeit der Öffentlichkeit erregen könnten. Eine solche Gefahr bestehe nicht bei deutschen Pilgern, die zusammen mit Bürgern anderer Staaten reisten. Internationale Regeln machten zudem die Zulassung von Konferenzteilnehmern unumgänglich, und die deutschen Spezialisten seien unersetzlich. ${ }^{95}$ Die Vorschriften deckten nicht alle Kategorien deutscher Besucher in Israel ab, lassen jedoch den Schluß zu, daß die persönliche Sicherheit der Besucher das wichtigste Entscheidungskriterium war. Die deutschen Matrosen auf deutschen Schiffen mit Schilumimgütern bereiteten der israelischen Polizei und anderen Sicherheitsorganen des Landes erhebliches Kopfzerbrechen. Doch die Befürchtungen bewahrheiteten sich nicht. Die Besatzungen der deutschen Schiffe stellten kein Sicherheitsrisiko dar, ganz im Gegensatz zu israelischen Matrosen, die in deutschen Häfen in Raufereien mit antisemitischem Hintergrund verwickelt waren. ${ }^{96}$

Als nicht unerheblich wurde das Sicherheitsrisiko erachtet, das von deutschen Flugpassagieren bei $Z$ wischenlandungen auf dem Flughafen Lod in Israel ausging. In solchen Fällen durften die Passagiere eine Nacht auf dem Flughafen verbringen und unbehelligt wieder ausreisen, trotz des Gesetzes zur Verfolgung von Nationalsozialisten. Der Besuch eines Vertreters des Auswärtigen Amtes in Israel auf einer Rundreise durch den Nahen Osten mußte im Jahr 1955 nach scharfen Protesten der Opposition in der Knesset abgesagt werden. Noch im selben Jahr reisten Friedrich Janz vom Bundeskanzleramt und Abraham Frowein vom Auswärtigen Amt nach Israel, ohne daß darüber Informationen an die Öffentlichkeit gelangten. ${ }^{97} \mathrm{Zu}$ den weiteren prominenten Besuchern zählte der Schilumimbeauftragte der SPD, der Bundestagsabgeordnete Heinrich Grewe, der evangelische Theologe Heinrich Grüber aus Berlin, Otto Küster, Böhms Partner bei den Verhandlungen

95 Najar, Westeuropaabteilung, an den Oberbefehlshabenden der israelischen Polizei vom 15. 5. 1954; Matam, Abteilungsleiter der israelischen Polizei, an den Direktor der Westeuropaabteilung vom 22. 5. 1954; Najar, Westeuropaabteilung, an den Oberbefehlshabenden der israelischen Polizei vom 23. 5. 1954, ISA, 2546/11.

96 Bericht vom 4. 8. 1952, ISA, 2542/11; HAARETZ (Tel Aviv) vom 5. 3. 1953; FAZ vom 15. 5. 1953; MaAriv (Tel Aviv) vom 19. 6. 1953; ZwISCHEN MORAL UND REALPOlITIK, Dok. Nr. 91, S. 327; ZMANIM (Tel Aviv) vom 5. 12. 1954.

97 Berichte vom 28. 9. 1955 und vom 9. 10. 1955, ISA, 590/6; Shinnar an den Stv. Leiter der Abt. Westeuropa vom 21.7. 1956, ISA, 2542/14. 
in Wassenaar, und Max Adenauer, der Sohn des Bundeskanzlers. ${ }^{98}$ Der israelische Geheimdienst forderte beim Holocaust-Forschungs- und Gedenkinstitut Yad Vashem Angaben über die Vergangenheit des jeweiligen Besuchers an.99

Deutsche Parteipolitiker, besonders SPD-Vertreter, waren häufige Gäste in Israel. Die meisten prominenten SPD-Bundestagsabgeordneten, unter ihnen auch Willi Eichler, Willy Brandt und Fritz Erler, begaben sich mindestens einmal nach Israel. Erich Ollenhauer stattete Israel 1957 einen Besuch ab, und Carlo Schmid folgte ihm zwei Jahre später. Schmid genoß als Politikwissenschaftler hohes Ansehen und wurde von der Hebräischen Universität mit einer Gastvorlesung geehrt. Seine Vorlesung war wahrscheinlich die erste in deutscher Sprache an einer israelischen Universität seit dem Krieg. ${ }^{100}$ Der Vorsitzende des Deutschen Gewerkschaftsbundes und andere führende westdeutsche Gewerkschafter besuchten Israel ebenfalls. Weit weniger häufig waren Besuche von CDU/CSU-Vertretern. Im Januar 1958 reiste Karl Graf von Spreti, der das Luxemburger Abkommen im Bundestag begründet hatte, nach Israel. Ihm folgten im Herbst 1962 der Präsident des Bundestages, Eugen Gerstenmaier, und im Frühjahr 1963 Franz Josef Strauß. Der Besuch von Strauß war von lautstarkem Protest der Linken begleitet. Frau Gerstenmaier nahm als Mitglied der bundesdeutschen Delegation an den Feierlichkeiten zum zehnjährigen Bestehen des Staates Israel teil. Sie wurde von Ben Gurion in Sdeh Boker, in der Negev-Wüste, empfangen. Mit Ausnahme von AltBundespräsident Theodor Heuss begab sich bis zu Thomas Dehlers Besuch im Jahr 1962 kein einziger hoher Vertreter der FDP nach Israel. Alt-Bundeskanzler Konrad Adenauer wurde 1966 in Israel sehr herzlich empfangen. Sein Besuch war aber auch von heftigen Studentenprotesten begleitet.

Wie bereits erwähnt, gingen von der Schilumimgesellschaft besonders viele Einladungen an offizielle Besucher aus Deutschland aus. Zu den Gästen der Schilumimgesellschaft gehörten neben Persönlichkeiten des öffentlichen Lebens und der Politik auch Geschäftspartner, deren Vergangenheit nicht immer über jeden Zweifel erhaben war. Der Direktor der Gesellschaft, Nahum Shamir, erinnerte sich später an seine diesbezüglichen Hemmungen. ${ }^{101}$ Die Schilumimgesellschaft kam für sämtliche Reise- und Aufenthaltskosten ihrer Gäste auf und zahlte ihnen in gewissen Fällen auch ein Taschengeld. Die Gesellschaft besorgte die Visa und organisierte Besichtigungstouren und Treffen mit Israelis.

98 Dawar (Tel Aviv) vom 20. 4. 1955; Duriel an Ilsar vom 20.11. 1955, ISA, 590/9; Shinnar an Sharett vom 24. 11. 1955; Shinnar an Ilsar vom 28. 2. 1956; Max Adenauer an Shinnar vom 20. 7. 1957, ISA, II, 183-12-chet; Tagebucheintrag Ben Gurions vom 7.11. 1958, BGA, BGD; MAARIV (Tel Aviv) vom 9. 3. 1956.

${ }^{99}$ Shinnar an die Abt. Westeuropa vom 18.6. 1954, ISA, 2542/13; Beratungen über Deutschland vom 13. 10.1955, ISA, 2539/4.

100 Shinnar an das Israelische Außenministerium vom 19. 9. 1956, ISA, 3099/25; Tagebucheintrag Ben Gurions vom 25.3. 1957; FAZ vom 22. 4. 1958; SCHMID, Erinnerungen, S. 637 ff.; Putzrath an Herlitz vom 15.7. 1960, AdsD, SPD-Vorstand; Shinnar an Brandt vom 31. 10. 1960, ISA, 3309/13.

101 SHAMIR, Interviews. 
In der Ausgabe von August 1957 kündigte der Israel-Informationsdienst eine Liberalisierung der Einreisebestimmungen für deutsche Touristen an. ${ }^{102} \mathrm{Bis}$ zu jenem Zeitpunkt reisten die wenigen deutschen Touristen, wie die Pilger, mit amerikanischen Gruppen in Israel ein. Um die Einreise ehemaliger Nationalsozialisten zu verhindern, mußten die Touristen eine Erklärung unterschreiben, wonach sie weder Mitglied der NSDAP gewesen waren, noch während des Krieges als Soldaten in der Wehrmacht gedient hatten. Rechtsgerichtete Knessetabgeordnete hielten dennoch an ihrer Opposition gegen den deutschen Tourismus in Israel fest, trotz sich daraus ergebender Einnahmen. Ein deutscher Spezialist für Israelreisen meinte im März 1961, es bestehe in der Bundesrepublik Interesse an Reisen nach Israel, warnte aber gleichzeitig vor einem zu schnellen Anstieg des deutschen Tourismus in Israel, da sich das Land zuerst daran gewöhnen müsse. ${ }^{103}$ Wie auch immer, die israelische Opposition gegen die Einreise von Deutschen verstummte nicht. Anläßlich eines internationalen Kongresses der Widerstandskämpfer und Opfer des Nazismus in Israel im Herbst 1958 gelang es israelischen Organisationen ehemaliger Widerstandskämpfer, die Teilnahme der deutschen Delegation, bestehend aus Franz Böhm und dem SPD-Bundestagsabgeordneten Alfred Frenzel, zu verhindern. 104

Auch das Auswärtige Amt war über den Anstieg des deutschen Tourismus in Israel im Hinblick auf negative arabische Reaktionen nicht glücklich. Die Situation gebe Anlaß zu „erheblichen Bedenken“, und die sich „häufenden“ Einladungen nach Israel würden ungute Reaktionen provozieren, hieß es. Einem Vertreter des Bundesfinanzministeriums wurde geraten, in Israel weder vor der Presse zu erscheinen noch mit Vertretern öffentlicher Organisationen zu sprechen. Er solle Zurückhaltung üben und sich nur zu vorab geplanten Treffen begeben. ${ }^{105}$ In Anbetracht der fehlenden diplomatischen Beziehungen könnten solche Reisen leicht mißverstanden werden, meinten Beamte des Auswärtigen Amts und wiesen darauf hin, daß in der arabischen Presse bereits "unliebsame" Kommentare erschienen seien. Außer Diplomaten, die unbeschränkt reisen dürften, gebe es zu viele offizielle deutsche Besucher in Israel, mit oder ohne Einladung. Einladungen nach Israel sei, wenn möglich, nur in Form von Privatbesuchen nachzukommen, empfahl das Auswärtige Amt. ${ }^{106}$ Die bundesdeutsche Diplomatie machte sodann Vertreter, die auf ein und derselben Reise einen Besuch in Israel und in arabischen Ländern planten, auf den arabischen Wirtschaftsboykott aufmerksam. ${ }^{107}$ Der

102 ISRAEL-INFORMATIONSDIENST Nr. 52 vom August 1957; MA'ARIV (Tel Aviv) vom 28. 6. 1957.

103 Denkschrift des Deutschen Bundesjugendringes vom März 1961, PA, 708, 82.22, 92.19.

104 Frankfurter AlLgemeine ZeITUNG vom 27. 10. 1958; interner Bericht von Alfred Frenzel über seine Eindrücke in Israel vom November 1958, AdsD, SPD-Vorstand, Abt. int. Beziehungen, Akte 2847.

105 Vermerk über die Reise des Herrn Reg. Dir. Koppe, BMF, vom 26. 3. 1959, PA, 708, 82.22, 92.19 .

106 Duckwitz, Abt. 7, an die Abt. 4 betr. die Einladung an Müller-Graff vom 30. 1. 1960, PA, 82.00, P92.19; vgl. ähnliche Schreiben und Vermerke vom 15. 2. 1961, 16. 1. 1962, 26. 4. 1960, 5. 9.1961, PA 708, 82.20, 92.19 .

107 Das Generalkonsulat, Damaskus, an das AA vom 17. 3. 1961, PA, 708, 82.20-82.70, 92.19. 
westdeutsche Tourismus in Israel hatte steigende Tendenz, und kein einziger deutscher Tourist wurde je abgewiesen. Die israelische Fluggesellschaft El Al war einer der großen Nutznießer dieses Reiseverkehrs. ${ }^{108}$

\section{Der Studenten- und Jugendaustausch}

Israelische Studenten entdeckten Deutschland schon sehr früh. Bereits im Frühjahr 1956 wurde der Aufenthalt israelischer Studenten in der Bundesrepublik vom israelischen Außenministerium als quantitativ bedeutende Erscheinung vermerkt. Aus den Dokumenten geht zudem hervor, daß die israelische Diplomatie von diesen Studenten erwartete, sich öffentlich und politisch für den Staat Israel einzusetzen. ${ }^{109}$ Es handelte sich um Emigranten aus Deutschland und um deren - fließend Deutsch sprechende - Nachkommen sowie um Einzelpersonen, die sich überwiegend auf bestimmte Fächer oder Berufe mit besonders guten Ausbildungsmöglichkeiten in Deutschland spezialisierten. Letztere hielten sich zum Teil aber auch aus anderen Gründen in Deutschland auf. Die Zahl der israelischen Studenten in Deutschland genügte 1956 offenbar für ein von deutschen Regierungsstellen organisiertes deutsch-israelisches Seminar, dessen Bedeutung für die israelische Öffentlichkeitsarbeit auch vom israelischen Außenministerium erkannt und deshalb unterstützt wurde. ${ }^{110} \mathrm{Zu}$ jener Zeit gab es keine offizielle Politik hinsichtlich Studienaufenthalten von israelischen Studenten in Deutschland. Studenten schrieben sich selbständig in deutschen Universitäten ein. Sie erhielten Stipendien und finanzielle Unterstützung von zu Hause. Die Abhängigkeit von solcher Unterstützung erforderte für Studien in Deutschland angesichts der Devisenbeschränkungen zwar ein gewisses Maß an behördlichem Entgegenkommen, doch verbindliche Vorschriften in diesem Bereich traten erst 1962 in Kraft. Danach war die Überweisung von Geldern ins Ausland für Studienzwecke nur an Studenten mit abgeschlossenem Militärdienst erlaubt und wenn das betreffende Studienfach in Israel nicht angeboten wurde und für die israelische Volkswirtschaft von Bedeutung war."11 Für Studien in anderen Ländern galten ähnliche Regeln, doch die speziellen Bestimmungen für Deutschland waren eindeutig darauf ausgelegt, Studenten vom Studium in diesem Land abzuhalten. 1968 schrieb sich eine deutsche Studentin an der Hebräischen Universität ein, um eine Dissertation über die Eingliederung von Einwanderern aus Deutschland in Israel zu schreiben. Die Presse betonte, daß ihr Vater Antifaschist gewesen sei. ${ }^{112}$

1958 fanden in der Bundesrepublik die ersten Sommeraustauschprogramme mit israelischer Beteiligung statt. Die wenigen israelischen Teilnehmer - vor allem

108 Vermerk vom 17. 1. 1962, PA, 708, 81.10/0-82.03, 92.19; DEUTSCHKRON, Israel und die Deutschen, S. 175.

109 Ilsar an Anug vom 25. 6. 1956, ISA, 613/7.

110 Ebd.; Shinnar und Anug an das israelische Außenministerium vom 1. 7. 1956; ISA, 613/7.

111 Vermerk der Informationsabteilung der Israel-Mission vom 10. 1. 1962, PA, 708, 82.03, 92.19; Israeli Official Print vom 11. 1. 1962, PA 708, 81.00-82.03, 92.19.

112 MA'ARIV (Tel Aviv) vom 8. 8. 1968. 
Wirtschafts- und Medizinstudenten - wurden sorgfältig ausgewählt. Besonders begehrt waren die von Studentenorganisationen und studentischen Reiseveranstaltern durchgeführten Programme bei Medizinstudenten, die entsprechenden Druck auf die Behörden ausübten. Die damals von der Cherut-Partei dominierte Studentenorganisation der Hebräischen Universität stimmte gegen den Studentenaustausch mit Deutschland. ${ }^{113}$ Das israelische Außenministerium berichtete, die Zusammenarbeit zwischen israelischen und deutschen Studenten stehe noch auf tönernen Füßen. 1956 prüfte Gertrud Luckner, ein führendes Mitglied des katholischen Caritas Verbandes aus Freiburg, in Israel die Möglichkeit, Israelreisen für deutsche Theologiestudenten zu organisieren. Doch das israelische Außenministerium war nicht begeistert. Man befürchtete eine kühle Aufnahme der deutschen Studenten. 1141957 berichtete der parlamentarisch-politische Pressedienst in Bonn über den Aufenthalt einer Gruppe von deutschen Studenten in Israel. ${ }^{115}$ Vor dem Hintergrund der allmählichen Verbesserung des Klimas in Israel organisierten die sowohl vom Bundesinnenministerium als auch vom Auswärtigen Amt unterstützten Deutsch-Israelischen Studiengruppen (DIS) 1959 Gruppenreisen nach Israel.116 Die Teilnehmer kehrten mit ausgezeichneten Eindrücken zurück. Sie zeigten sich überzeugt von der Notwendigkeit der Bekämpfung des Antisemitismus, und einzig die Lage der arabischen Bevölkerung in Israel gab zu Kritik Anlaß.117 Das Auswärtige Amt sympathisierte mit dem Studentenaustausch, zögerte aber mit finanzieller Unterstützung. ${ }^{118}$ Die israelische Rechte stand diesen Studentenreisen kritisch gegenüber, konnte sie aber nicht mehr verhindern.

Der Jugendgruppenreiseverkehr von und nach Israel begann Mitte der fünfziger Jahre und entwickelte sich ab Mitte der sechziger Jahre zu einer wahren Massenbewegung. Die Anfänge werden unterschiedlich beschrieben. Nach Ralph Giordano begann der Jugendreiseverkehr mit dem Treffen des internationalen Jugendherbergsverbandes von 1950 in London. Die erste deutsche Jugendgruppe reiste dann, laut Giordano, 1957 nach Israel. ${ }^{119}$ Einer anderen Quelle zufolge soll der ursprüngliche Impuls von der Kölner Stadtverwaltung ausgegangen sein, die 1959 eine erste Gruppe von Jugendlichen nach Israel entsandte. 120 Der Jugendgruppenreiseverkehr genoß die Unterstützung der Arbeitsgemeinschaft für Jugendliche und Jugendfürsorge, deren Tätigkeit auch beim Auswärtigen Amt auf Anerkennung stieß. ${ }^{121}$ Der Hauptgeschäftsführer des Deutschen Bundesjugendrings, Heinz Westphal, reichte dem Auswärtigen Amt im März 1960 eine ausführliche Denkschrift zum Thema Jugendaustausch ein. ${ }^{122}$ Westphal stellte ein wach-

113 Savir an Shinnar vom 24. 5. 1959, ISA, 300/7.

114 Ilsar an Shinnar vom 8. 2. 1956, ISA, 590/9.

115 Der Parlamentarisch-Politische Pressedienst vom 4. 11. 1957, ISA, 3099/25.

116 Hess an die Westeuropaabteilung vom 9. 4. 1959, ISA, 3100/10II; Savir an Shinnar vom 24. 5. 1959, ISA 300/7; Voigt an das Ref. 604 vom 10. 2. 1960, PA, 1035, 708, 82.60, 92.19.

117 TAgESSPIEgEL vom 25.11. 1960.

118 Ref. 708 an das Ref. 603 vom 13. 7. 1960, PA, 1035, 708, 82.60, 92.19.

119 NARBEN, SPUREN, ZEUGEN, S. 414.

120 Ref. 708 an Ref. 603 vom 13.7.1960, PA, 1035, 708, 82.60, 92.19.

121 Kolb, Ref. 604, an das Ref. 708 vom 13. 3. 1959, PA, 708, 82.22.

122 Denkschrift des Deutschen Bundesjugendringes betr. Prinzipielle Überlegungen zur Ent- 
sendes Interesse bei deutschen Jugendlichen am Kontakt zu Israelis und zu israelischen Jugendlichen fest. Auch die israelische Jugend sei an Treffen mit Jugendlichen aus anderen Ländern interessiert, wenn auch die Begegnung mit deutschen Jugendlichen, so Westphal, nicht ganz unproblematisch sei. Nur wenige Organisationen in Israel seien bereit, schrieb Westphal weiter, deutsche Gruppen aufzunehmen und durchs Land zu führen. Die deutschen Jugendlichen würden sich in der Regel eine Weile in Kibbuzim aufhalten, wenn auch bislang nur der Mapai angegliederte Kibbuzim bereit gewesen seien, die jungen Leute aufzunehmen. Westphal hielt fest:

„Der Grundgedanke für die Entsendung deutscher Reisegruppen nach Israel kann es heute nur sein, eine intensive menschliche Begegnung herbeizuführen, zur Aussprache über Vergangenheit und Gegenwart zur Verfügung zu stehen, durch das eigene Verhalten den Nachweis zu führen, daß in unserem Land eine neue Generation heranwächst, die entschlossen ist, einen demokratischen Weg zu gehen und Unrecht wiedergutzumachen. Dazu kommt das Kennenlernen der Aufbauleistung des israelischen Volkes, das Studium der Wege, die dabei beschritten worden sind." 123

Solche Reisen waren mit speziellen Vorbereitungen verbunden. Dazu gehörte auch der Unterricht über die jüngste deutsche Vergangenheit und den Holocaust sowie über die Gegenwartsgeschichte des jüdischen Volkes, des Staates Israel und des israelisch-arabischen Konflikts. Das Aufenthaltsprogramm der Gruppen in Israel läßt auf eine sehr sorgfältige Planung schließen. Die Jugendlichen wurden auch aufgefordert, den Kontakt mit Israelis zu suchen. Die Finanzierung der Reisen erfolgte aus eigenen Mitteln mit Zuschüssen der Regierung und öffentlicher Institutionen. ${ }^{124}$

Die Reisen nach Israel wurden von Jugendlichen in der Bundesrepublik, wie bereits dargelegt, mit großem Enthusiasmus, Interesse und Neugier erwartet. Zum Teil wurden solche Reisen als eine Art Sühne für die NS-Verbrechen betrachtet. Besonders ausgeprägt zeigte sich dies nach der Swastikawelle Anfang 1960. Sie führte später zur Gründung der Aktion Sühnezeichen/Friedensdienste. ${ }^{125}$

Auch das Jugendherbergswerk trug zur Förderung der Jugendreisen bei. Gönner aus Deutschland leisteten einen Beitrag zum Bau von Jugendherbergen in Israel, die jeweils nach ausgelöschten jüdischen Gemeinden oder im Holocaust umgekommenen jüdischen Persönlichkeiten benannt wurden. ${ }^{126}$ Die deutschen Jugendgruppen übernachteten in diesen Jugendherbergen, die zum Teil mit direkter Hilfe der deutschen Jugend errichtet worden waren. Das israelische Außenministerium verfolgte die Israelreisen deutscher Jugendlicher mit einer gewissen Reserve und betrachtete die Annäherung zwischen Jugendlichen beider Länder

sendung deutscher Reisegruppen nach Israel und damit im Zusammenhang stehende Fra123 Ebd. gen vom März 1961, PA, 708, 82.22, 92.19.

124 Ebd.; HAASE, 20 Jahre; Varon an Ernst Simon vom 17. 1. 1961, ISA, 3309/14; KNESSETPROTOKOLle [hebr.], 136. Sitzung am 30. 5. 1962, S. 2100.

125 BÖHME, Die Arbeit der "Aktion Sühnezeichen".

126 KNESSET-PROTOKOLLE [hebr.], 258. Sitzung am 20. 3. 1961. 
mit Skepsis. Der Vorschlag Heinrich Grübers, einen Ausschuß zur Förderung der Beziehungen zwischen Jugendlichen beider Länder einzusetzen, wurde vom israelischen Außenministerium umgehend verworfen. Israel, hieß es in einem internen Papier, sei daran zur Zeit nicht interessiert. Grüber sei mitzuteilen, daß gegenwärtig in Anbetracht der deutschfeindlichen Stimmung im Land kein Spielraum für den Jugendaustausch bestehe. Statt dessen konzentriere man sich besser auf die Einladung verdienstvoller Persönlichkeiten.127

Im März 1959 bat Teddy Kollek, einer von Ben Gurions Assistenten und späterer Bürgermeister von Jerusalem, seinen Chef um Erlaubnis, eine israelische Jugenddelegation zum Jugendherbergskongreß in Deutschland zu entsenden. Ben Gurion lehnte ab. „Wir werden keine Jugendgruppen dorthin schicken“, notierte er in sein Tagebuch. ${ }^{128}$ Deutsche Jugendliche wären gerne bereit gewesen, israelische Jugendliche zu empfangen. ${ }^{129}$ Die deutsche Seite zeigte Verständnis für die israelischen Hemmungen („aus [...] ausgesprochenen Gründen kann von Israel nicht erwartet werden, daß deutsche Gegeneinladungen schon jetzt angenommen und befolgt werden. Dafür gilt es Verständnis zu haben "130), gab aber gleichzeitig der Hoffnung Ausdruck, sie könnten bald überwunden werden. Ein erstes Anzeichen dafür war die Deutschlandreise einer Mapai-Jugendgruppe im Jahre 1961, die vom israelischen Außenministerium unter der Bedingung bewilligt wurde, daß daran keine Jugendlichen im Schulalter teilnehmen würden. ${ }^{131}$ Die für den Kontakt mit deutschen Gewerkschaften zuständige israelische Gewerkschafterin Yehudith Simchoni bemerkte zu einer Einladung von einunddreißig jungen israelischen Gewerkschaftern nach Berlin: „Ich bin zwar grundsätzlich für die Verbreitung von Information über Israel und die Förderung von Kontakten mit jüngeren Bevölkerungsschichten in Deutschland, kann aber persönlich nicht daran teilnehmen. "132 Während viele junge Israelis einer Reise nach Deutschland nicht abgeneigt waren, prüften Vertreter der älteren Generation zuerst jedes Detail über die Gastgeber und versuchten, die Annäherung zu bremsen. ${ }^{133}$ Die Israelis erwarteten von ihren Gastgebern zudem die Deckung der Reise- und Aufenthaltskosten, eine schwere Last für die Gastgeber. ${ }^{134}$ Obwohl immer mehr israelische Jugendliche an Gruppenreisen nach Deutschland teilnahmen, waren noch längst nicht alle Probleme des Jugendaustausches ausgeräumt.

Der informelle Kontakt zwischen der Bundesrepublik und Israel war vermutlich der beste Seismograph des dynamischen deutsch-israelischen Verhältnisses.

127 Varon an Shinnar vom 10. 8. 1961, ISA, 3309/4.

128 Tagebucheintrag Ben Gurions von einem nicht näher bezeichneten Tag im Jahr 1959, BGA, BGD.

129 Savir an Wolfgang W. Rücker, Johanniter-Unfall-Hilfe, vom 27. 5. 1964, ISA, 3532/4.

130 Denkschrift des Deutschen Bundesjugendringes betr. Prinzipielle Überlegungen zur Entsendung deutscher Reisegruppen nach Israel und damit im Zusammenhang stehende Fragen vom März 1961, PA, 708, 82.22, 92.19.

131 Westeuropaabteilung an Brosch vom 15. 12. 1961, ISA, 3309/14.

132 Yehudith Simchoni, Exekutivrat der Histadrut, an Zeev Scheck, Direktor der Abteilung für Internationale Beziehungen des israelischen Außenministeriums, vom 4.3. 1965, ILA, Group 291a, File 132b.

133 HaARETZ (Tel Aviv) vom 17. 8. 1965.

134 HAASE, 20 Jahre, S. 99-100. 
Während offizielle israelische Stellen die Annäherung zu bremsen versuchten, dominierte in manchen Bereichen - wenn auch in sehr unterschiedlichem $\mathrm{Maß}$ - auf beiden Seiten der Wunsch, die gesellschaftlichen und kulturellen Beziehungen zu vertiefen. Insgesamt war eine allmähliche Lockerung kontaktfeindlicher Gesetze und Verordnungen und ein wachsendes Interesse am gegenseitigen Austausch festzustellen. Die Außenministerien beider Länder verdienen dagegen schlechte Zensuren. Sie versuchten, die von der Bevölkerung ausgehende Dynamik der Annäherung zu bremsen bzw. in kontrollierbare Bahnen zu lenken. Je weniger die Ämter involviert waren, desto schneller kam die Annäherung voran. Mit anderen Worten, je mehr die behördliche Kontrolle zurückging, desto mehr nahm die Neigung auf israelischen Seite zu, die Selbstbeschränkungen im Hinblick auf Kontakte mit Deutschland fallenzulassen, und um so mehr verstärkte sich auch der Wunsch auf deutscher Seite, die Annäherung zu Israel zu fördern. Die Versuche beider Regierungen, die informellen Beziehungen zu hemmen, blieben letztlich erfolglos und waren sogar kontraproduktiv. Im Gegensatz zum gewundenen Weg zur Aufnahme diplomatischer Beziehungen verlief die informelle Annäherung zwischen beiden Ländern in geraderen Bahnen und überwiegend in positiver Richtung. 
